\title{
Computational investigation on a self-propelled pufferfish driven by multiple fins
}

\author{
Ruoxin $\mathrm{Li}^{1}$, Qing Xiao ${ }^{1 *}$, Yuanchuan $\mathrm{Liu}^{2}$, Lijun $\mathrm{Li}^{3}$, Hao $\mathrm{Liu}^{3,4}$
}

1. Department of Naval Architecture, Ocean and Marine Engineering, University of Strathclyde, Glasgow, UK

2. College of Engineering, Ocean University of China, Qingdao 266100, China

3. Shanghai Jiao Tong University and Chiba University International Cooperative Research Centre (SJTU-CU ICRC), Shanghai Jiao Tong University, Shanghai, China

4. Graduate School of Engineering, Chiba University, Chiba, Japan

Corresponding author: qing.xiao@strath.ac.uk

\begin{abstract}
Based on experimental measurements of a live fish, a numerical model is established for a pufferfish driven by the locomotion of its multiple flexible fins. The self-propelled motion of the fish is investigated under unsteady swimming conditions, including the accelerating and subsequent quasi-steady stage, to analyse the motion mechanisms of a real fish model with multiple flexible fins. Present numerical approach combines Computational Fluid Dynamics (CFD) and Multi-Body Dynamics (MBD), which allows for the analysis of the interaction between fluid and a fish with multiple fins. Benefitting from the available experimental data of the live fish, the deformation of each fin surface can be prescribed, and we name this condition as flexible in this paper. To elucidate the impact of flexible fins on the propulsion performance of fish swimming, simulations are also performed for rigid fins with the same kinematic motion profiles imposed on the leading edge of fin surfaces. With the developed tool, unsteady hydrodynamic forces, vortex interaction between body and fins associated with MPF swimming mode are numerically predicted. The obtained results highlight the effect of flexibility of fins on thrust generation and efficiency improvement for fish undergoing free-swimming.
\end{abstract}

Keywords: self-propelled, Median-Paired Fin (MPF) mode, pufferfish, multiple fins, Multi-Body Dynamics, Computational Fluid Dynamics 


\section{Introduction}

Along with the substantial increase in ocean exploration, Autonomous Unmanned Vehicles (AUV) have played a significant role in underwater tasks, such as aquatic environment surveys, oil spill examinations, offshore structure fatigue checks (Blidberg, 2001; Salazar et al., 2018). Some bio-inspired AUV systems adopt the morphology and swimming mechanisms of aquatic animals, especially fish, to enable such devices with extraordinary propulsion and manoeuvring abilities (Ijspeert, 2014; Scaradozzi et al., 2017).

Swimming modes for fish are generally categorized into two types, i.e. Body-Caudal Fin (BCF) and Median-Paired Fin (MPF) (Lindsey, 1978; Webb, 1984). Unlike the BCF mode, where the generation of propulsion thrust is achieved via bending fish body into a backward travelling wave from the fish head to the caudal fin, an evident feature for the MPF swimming mode is that a swimmer of this type employs its multiple fins' motions (such as caudal, pectoral, dorsal and anal fins) for manoeuvring and propulsion.

Many researches have been carried out to study the interactions between multiple fins. There have been some experimental studies of live fish with the Particle Image Velocimetry (PIV) method. Drucker and Lauder (2001) investigated the interaction between dorsal and caudal fins. Their results revealed that the vortices generated by the upstream dorsal fin could interact with those generated by the downstream caudal fin. The study of Standen and Lauder (2007) was extended from dorsal and caudal fins to include an anal fin. The flow around the caudal fin was found to be greatly affected by the existence of both dorsal and anal fins. Tytell (2006) pointed out that the threedimensional vortex wake structure associated with a caudal fin was linked up with those generated by the dorsal and anal fins. Extensive studies have also been carried out using numerical methods such as Computational Fluid Dynamics (CFD) and focused on the flow and vortex generation mechanisms for fin-fin interactions. As an initially simplified step, the morphology of fins was modelled as a flat plate while the fish body was ignored. It is illustrated that, in a tandem configuration, the vortices generated by the upstream plate travel backward and interact with the downstream one, influencing its thrust generation and the overall propulsion efficiency (Broering and Lian, 2012; Kourosh and Qiang, 2015). 
In recent years, more realistic fish models with a fish body and multiple fins have been investigated in CFD simulations under either tethered-swimming (a constant velocity of the incoming flow is specified and the fish is not allowed to move freely) or freeswimming (the fish can move freely) conditions. The geometry and kinematics of a three-dimensional fish can be obtained from experimental measurements. In some studies, dorsal and anal fins were geometrically included in the fish model while the fins only moved along with the fish body without considering their individual kinematic motions (Borazjani, 2013; Borazjani et al., 2012; Li et al., 2016). Liu et al. (2017) studied a carangiform fish under the tethered-swimming condition with three established CFD models, given the name of M1 (fish body with dorsal, anal and caudal fins), M2 (body-caudal fin) and M3 (caudal fin only). Comparing results from all three models, they concluded that the drag force of the fish body can be reduced if the interaction among all three fins was taken into account even though the individual motions of anal and dorsal fins were not numerically modelled in M1. Yu et al. (2011) investigated the tethered swimming of a model with an undulating fish body and a pair of fixed pectoral fins. Results revealed that a pair of vortices could be formed immediately behind the pectoral fins and it can interact with and the undulating fish body. More sophisticated studies have also been carried out, taking into account individual fin motions in their fish models. Xu and Wan (2012) studied the selfpropelled motion of a fish with a pair of rigid pectoral fins while flapping and feathering motions of the fins were considered. It was found that adjusting parameters of fin motions would change the forces generated by the fins and consequently the fish motion.

For real fish swimming, the fins of a fish not only have individual motions but also are flexible/deformable rather than rigid, i.e. the shape of a fin can change during swimming. Previous experimental findings on live fish suggested that the fin rays of a fish had different kinematic locomotion parameters (Lauder and Madden, 2007; Li et al., 2018a). A number of numerical studies have also been carried out to investigate the role of flexible fins on fish swimming behaviour, as has been reviewed comprehensively by Wang et al. (2016). By simplifying a fin model as a thick plate, it was noted that the hydrodynamic performance of a fin with appropriate flexible stiffness was better than that of a rigid fin (Kim et al., 2016; Park and Sung, 2018). Liu et al. (2018) investigated the hydrodynamic performance of both rigid and flexible foils in a wave environment. It was noted that comparing to a rigid foil, a flexible one could 
achieve a larger thrust force and a higher propulsive efficiency. Zhu and Shoele (2008) examined the flexibility effect of a caudal fin by modelling the passive deformation of fin rays with a number of uniform Euler-Bernoulli beams. Subsequent studies were extended to a skeleton-strengthened pectoral fin (Shoele and Zhu, 2009, 2010). Their predicted results revealed that the thrust and propulsion efficiency increased as a result of the passive deformation of fin rays. Zhou et al. (2017) further studied a fish bodycaudal fin model and revealed that a faster swimming speed could be achieved for a fish model with a flexible caudal fin than that with a rigid one. It can thus be summarised from the above literature reviews that it is necessary to take into consideration the change in the fin shape while studying fish swimming behaviour. To the best of the authors' knowledge, rare work has been done to study the hydrodynamics of a self-propelled fish with multiple flexible fins using a numerical approach.

The present study considers a free-swimming pufferfish driven by its dorsal, anal and caudal fins (see the supplementary movie online). One notable feature of our model is that all fins have different undulating profiles, which were extracted from a live fish experiment ( $\mathrm{Li}$ et al., 2018a). Prescribing individual surface motions to each fin allows us to replicate the deformation of real fish fins, and we name this condition as flexible. Additionally, the locomotion of the fish in the present study is purely driven by the motions of its fins and induced by the interactions between the fish and surrounding fluid. The objective of this study is to analyse the impacts of the deformation of multiple fins on self-propelled fish swimming. A corresponding rigid condition is thus also simulated as comparison, where the motion of the whole fin surface is uniform. To solve the dynamics of the fish, our in-house code is used, which is based on the Multi-Body Dynamics (MBD) theory (Porez et al., 2014b). This MBD code was established to analyse the self-propelled motions for various types of BCF swimmers $(\mathrm{Hu}, 2016)$ and has been recently extended to simulate fish of the MPF mode (Li et al., 2018b), with the ability to deal with motions of both rigid and flexible fins. Numerical simulations are carried out using a Computational Fluid Dynamics (CFD) method. The commercial software ANSYS Fluent 15.0 is employed to solve fluid flow. 


\section{Numerical model}

\subsection{Description of a Pufferfish Body-Caudal-Dorsal-Anal Model}

Pufferfish is a typical MPF Tetradontiform swimmer whose pectoral, dorsal anal and caudal fins could mobilize independently (Blake and Chan, 2011; Gordon et al., 1996). Fig. 1(a) and (b) show a vertical and a side view of the pufferfish measured in the experiment ( $\mathrm{Li}$ et al., 2018a). A 3D model is extracted and employed in the present numerical study, as sketched in Fig. 1(c). The total length $(L)$ of the model is approximately $0.11 \mathrm{~m}$. The shape of each cross-section of the body is approximated as an ellipse. The width $W$ and height $H$ of the model are approximately 0.04 and $0.03 \mathrm{~m}$, respectively. Each of the three fish fins is modelled as a wedged body surface and the thickness of a fin gradually decreases to zero from the root to the edges including tip, leading and trailing fin rays (as shown in Fig. 2).

The live fish experiment was conducted in a circulating water tank with a controllable constant incoming current speed. High-resolution video cameras were used to record the dynamic responses of the fish and the surface motions of its fins. It was found in the experiment that pectoral fins adhered firmly to the main fish body without any oscillations ( $\mathrm{Li}$ et al., 2018a). Therefore, the impact of the pectoral fins on the swimming behaviour is not numerically modelled in this numerical study. The fish body is modelled as rigid and pectoral fins are omitted. Fig. 1(d) displays the experimental captures at four different instantaneous time in one motion cycle, indicating the profound undulating motions of the dorsal, anal and caudal fins. Subsequently, we impose the obtained kinematics of each fin surface into our model as the prescribed fin deformation and define this condition as flexible, which is different from the conventionally accepted passively deformed fins. For each fin surface in the experiment, the motions of seven fin rays were tracked as illustrated in Fig. 1(e). In the present CFD study, each fin surface could be considered as a series of successive fin rays. Driven by the leading fin ray, a sinusoidal wave travels from the anterior-most edge and spreads down to the rest of the fin surface. By interpolating the motions of those tracked fin rays in the experiment, the motion of the entire fin surface could be approximately obtained and subsequently prescribed in CFD simulations as the flexible condition. 
The kinematics of the flexible fins measured in the experiment under two velocity conditions are tested in this work, i.e. $1 B L / s$ and $2 B L / s$, where $B L$ is the body length of the pufferfish. Theoretically, the predicted cruising velocity of a self-propelled pufferfish in an initially still water environment should be equal to the incoming current speed given in the experiment testing if the kinematic motion profiles of the CFD model are extracted from the experiment live fish. To analyse the effects of flexible fins in relation to fish propulsion, the rigid fins for the same pufferfish model are also simulated. Table 1 summarises the main motion parameters for both rigid and flexible conditions. For the flexible fins, the motion profiles of Case 2 and Case 4 are taken from the experimental data associated with a current velocity of $1 B L / s$ and $2 B L / s$. Fig. 2 shows the sketches of leading, trailing fin rays and fin tips for dorsal, anal and caudal fins.

The kinematic equation to describe the surface motion of a flexible fin is expressed as:

$$
\varphi(\theta, t)=A(\theta) \sin (\omega t+\psi(\theta))
$$

where $\theta$ is the angle between a fin ray (represented by a blue line in Fig. 2) and x axis relative to its local coordinate (red lines in Fig. 2); $A(\theta)$ and $\psi(\theta)$ are the amplitude and phase angle for the fin ray, respectively; $\omega$ is the flapping frequency and changes for different cases. By varying $A(\theta)$ and $\psi(\theta)$, the deformation of flexible fin surface over one motion period is shown in the left column of Fig. 3. For dorsal and anal fins, the amplitude $A(\theta)$ and phase angle $\psi(\theta)$ can be expressed as:

$$
\begin{aligned}
& A(\theta)=a_{I} \theta^{3}+a_{I I} \theta^{2}+a_{I I I} \theta+a_{I V} \\
& \psi(\theta)=p_{I} \theta^{3}+p_{I I} \theta^{2}+p_{I I I} \theta+p_{I V}
\end{aligned}
$$

The prescribed surface motion of a flexible caudal fin can be defined as:

$$
\begin{aligned}
& A_{c}(\theta)=a_{I}+a_{I I} \cos \left(\theta \omega_{a}\right) \\
& \psi_{c}(\theta)=p_{I}+p_{I I} \cos \left(\theta \omega_{p}\right)
\end{aligned}
$$

Values for the parameters in Eqs. (2)-(3) can be found in Table 1.

A rigid-fin model is also set up as Case 1 and Case 3 in Table 1. As all flexible fins are driven by their leading fin rays (Li et al., 2018a), the amplitudes of the leading fin rays 
for rigid fins are set to be identical to their flexible counterparts to ensure the results are comparable. It was noted from the experiment that the dorsal and anal fins undulated in phase with each other, while there was a 180-degree $(\pi)$ phase lag between the caudal fin and the other two fins. The motion profile is mathematically given in Eq. (4) and graphically plotted in Fig. 3 for Case 4 (left, flexible) and Case 3 (right, rigid), where the shape envelopes of each fin at ten evenly distributed times are displayed in one motion cycle.

$$
\varphi(t)=A \sin (\omega t+\psi)
$$

where $\omega$ is the flapping frequency; $\psi$ is the phase lag and $A$ the flapping amplitude. Typical values for $\omega, \psi$ and $A$ can be found in Table 1 .

\subsection{CFD method and MBD method}

In the present study, the commercial software ANSYS Fluent 15.0 is used to solve the fluid flow around a swimming fish with the pressure-based transient solver. The governing equations are the three-dimensional incompressible continuity and momentum equations as follows:

$$
\begin{aligned}
& \nabla \cdot \vec{u}=0 \\
& \frac{\partial \vec{u}}{\partial t}+(\vec{u} \cdot \nabla) \vec{u}=-\frac{1}{\rho} \nabla p+\frac{\mu}{\rho} \nabla^{2} \vec{u}
\end{aligned}
$$

where $\vec{u}=(u, v, w)$ is the fluid velocity vector, $p$ is the fluid pressure, $\mu$ is the fluid viscosity and $\rho$ is the fluid density. A first order implicit time marching scheme is adopted for the transient terms. The second-order upwind scheme is employed for diffusive term discretization. Pressure-Velocity coupling is achieved by enabling the Non-Iterative Time Advancement (NITA) scheme and the Fractional Step Method (FSM). The density of the fish model is assumed the same as that of water, $\rho_{\text {fish }}=\rho_{\text {water }}$, which is a reasonable assumption for aquatic animals and thus the influence of gravity and buoyancy is ignored.

As the swimming of the pufferfish is fully induced by the motions of rigid/flexible median fins, the motion of the fish is solved by adopting a Multi-Body Dynamics (MBD) algorithm implemented as a User Defined Function (UDF) in ANSYS Fluent. Detailed 
information about the MBD algorithm has been explained and validated in our previous paper (Li et al., 2018b). By adopting the MBD concept, the CFD pufferfish model can be considered as a tree-structured model with four "bodies/elements" and virtual hinges connecting two successive elements as shown in Fig. 4. To solve this problem, in addition to an earth or global coordinate, a local coordinate is defined for each element. A reference element $B_{0}$ is selected to represent the key parameters of the fish model, such as swimming velocity, position and orientation relative to earth coordinate. In the present study, the fish body, coloured in yellow in Fig. 4, is selected as the reference element $B_{0}$ and the dorsal, anal and caudal fins are three different branches numbered as $B_{1}, B_{2}$ and $B_{3}$.

\subsection{Solution procedure of fish motion}

At each time step, a data transfer process occurs between the fluid solver and our inhouse MBD code. Detailed coupling for this fluid-structure interaction problem is as follows:

1. Update the prescribed kinematic motion of fish fins at the beginning of each time step as described in Section 2.1.

2. Calculate hydrodynamic force $f_{\text {ext }, i}$ of each element using the fluid solver. At each time step, the fluid force and moment exerted upon each element are integrated over its surface and then transferred to the MBD solver.

3. Transfer fluid force on each element to the reference element $B_{0}$. The forces on fins are transferred backwards to the reference element, i.e. fish body $B_{0}$, by using the following equations:

$$
\begin{aligned}
\text { for } i & =1,2,3 \\
\beta_{i}^{*} & =\beta_{i}-f_{e x t, i} \\
\beta_{0}^{*} & =\left(\beta_{0}-f_{e x t, 0}\right)+\sum_{i} A d_{g_{g_{i}}}^{T}\left(\mathrm{M}_{i}^{*}\left(A \ddot{\varphi}_{i}+\varsigma_{i}\right)+\beta_{i}^{*}\right) \\
\mathrm{M}_{0}^{*} & =\mathrm{M}_{0}+\sum_{i} A d_{{ }_{g_{i}}^{T}} \mathrm{M}_{i} A d_{0_{g_{i}}}
\end{aligned}
$$

where $\beta_{i}$ is a $(6 \times 1)$ vector for Coriolis and centrifugal forces of the $i$ th element. $i=1,2,3$ stands for the three elements in this multibody dynamic model, i.e. dorsal, 
anal and caudal fins. The variable $A d_{0_{g_{i}}}^{T}$ is the transpose matrix of an adjoint map operator $A d_{i_{g_{0}}}$, and stands for the transformation matrix for mass and force from the $n$th coordinate to the coordinate of reference element $B_{0} . \mathrm{M}_{i}$ is the inertia tensor, which includes the element mass $M_{i}$, the first inertia moment $M \hat{S}_{i}$ and angular inertia $I_{i}$ as:

$$
\mathrm{M}_{i}=\left(\begin{array}{cc}
M_{i} & -M \hat{S}_{i} \\
M \hat{S}_{i} & I_{i}
\end{array}\right)
$$

$\varsigma_{i}$ stands for a partial acceleration (Porez et al., 2014a). $A$ is a $(6 \times 1)$ unit vector and $\ddot{\varphi}_{i}$ is the angular acceleration of the $n$th element.

4. Obtain the acceleration $\dot{\eta}_{0}$. To compute the motions of the self-propelled fish at each time step, Newton's second law is applied. The acceleration $\dot{\eta}_{0}$ of reference element $B_{0}$ can be calculated as:

$$
\dot{\eta}_{0}=-\left(\mathrm{M}_{0}^{*}\right)^{-1} \beta_{0}^{*}
$$

5. Determine the velocity ${ }^{e} \eta_{i}$ for each element and transfer motion data to the fluid solver. After obtaining the acceleration $\dot{\eta}_{0}$ from Equation (8), the velocity $\eta_{0}$ for the reference element, a $(6 \times 1)$ vector which contains linear and angular velocity in three dimensions, can be calculated by using a 4th order Runge-Kutta integration as:

$$
\left.\eta_{0}\right|_{t+1}=\left.\eta_{0}\right|_{t}+\frac{\Delta t}{6}\left(\left.\dot{\eta}_{0}\right|_{t} ^{1}+\left.2 \dot{\eta}_{0}\right|_{t+\frac{\Delta t}{2}} ^{2}+\left.2 \dot{\eta}_{0}\right|_{t+\frac{\Delta t}{2}} ^{3}+\left.\dot{\eta}_{0}\right|_{t+\Delta t} ^{3}\right)
$$

Once $\eta_{0}$ is estimated, the velocity of other bodies in their local coordinates can be determined in the following way:

$$
\eta_{i}=A d_{i_{0}}{ }^{e} \eta_{0}+\dot{\varphi}_{i} A \quad i=1,2,3
$$

Here, the angular velocity $\dot{\varphi}_{i}$ is the time derivative of the angular motion $\varphi_{i}$. Velocity for all elements relative to earth coordinate can be obtained as:

$$
{ }^{e} \eta_{i}=\left(\begin{array}{cc}
{ }^{e} R_{i} & 0 \\
0 & { }^{e} R_{i}
\end{array}\right) \eta_{i}
$$


The velocity component of reference element ${ }^{e} \eta_{0}$ in $\mathrm{X}$ direction is denoted as “induced velocity $U$ " for the whole fish system. Newly updated velocity ${ }^{e} \eta_{i}$ for each element is sent back to fluid solver for the calculation in the next time step.

6. Repeat steps 1-4 for all time marching steps during a coupled simulation.

\subsection{Computational domain and boundary conditions}

The computational domain is generated using a cylindrical topology and separated into inner and outer zones, as displayed schematically in Fig. 5. The length and the radius of the overall computational domain are $12 L$ and $5 W$, where $L$ and $W$ are the length and width of the fish model. As the fish swims towards the inlet boundary, the distance from the fish to the inlet is set to be larger than the distance away from the outlet. The outer zone is designed wide enough to avoid the influence of outer boundaries, and a relatively coarse mesh is adopted to achieve a reasonable mesh number. Meanwhile, as the self-propelled pufferfish model swims mainly in the inner cylindrical zone with a dimension of $10 L \times 3 W$, the mesh is deliberately refined in the inner zone to better capture flow characteristics and ensure the accuracy of results.

Unstructured tetrahedral elements are used in the computational domain. The upstream and surrounding boundaries are set as velocity inlet, i.e. $\vec{u}=(0,0,0)$. The downstream boundary is set as pressure outlet with a zero gauge pressure. A no slip boundary condition is applied to the surface of fish. Since fish swim in still water, the initial flow field velocity is set to zero.

\subsection{Grid and time step size independence tests}

Due to the large deformation of the mesh when the fish swims, the dynamic mesh functionality available in FLUENT is used. In order to guarantee the mesh quality during each time step, smoothing and re-meshing functions are employed.

To quantify the dependence of the numerical results on the mesh density, a simulation of a free-swimming pufferfish with the kinematics in Case 4 is performed. Three sets of mesh with different grid density are generated as summarised in Table 2, in which $N_{\text {face }}, N_{\text {cell }}, \Delta h_{\text {min }} / L$ and $\Delta h_{\text {max }} / L$ represent the number of faces on the surface grid of the fish, the number of mesh cells in the computational domain, the minimum and 
maximum dimensionless cell height, respectively. The cell height is normalized by the fish length $L$. Total mesh number of the medium mesh is 1.6 million, which is about three times of the coarse mesh and half of the fine one. Fig. 6 (a) shows the results of grid independence tests in the 29th undulating period with respect to total force $F_{T}$. It illustrates that $F_{T}$ obtained from the medium mesh is almost identical to results from the fine mesh but is slightly different from that with the coarse one. Meanwhile, as the grid around fins are deformed greatly during fish swimming, force results around the dorsal fin from the mesh independence test are also compared in Fig. 6(b). It is shown that the time series of force obtained with medium and fine mesh density are almost the same while the result from the coarse mesh is noticeably smaller than the other two cases. Hence the medium mesh is selected for all simulations.

As it is an unsteady problem, a time-step size dependence test is also conducted with the medium mesh using the kinematics of flexible fins in Case 2. Three time-step sizes are selected namely $\Delta t=T / 250, T / 500, T / 750$, where $\mathrm{T}$ stands for the motion period $(T=2 \pi / \omega)$. Corresponding results are given in Fig. 6 (c), presenting the total force $F_{T}$ on the fish in the $29^{\text {th }}$ motion cycle. The results are almost identical between $\Delta t=T / 500$ and $\Delta t=T / 750$, and a slight difference exists for $\Delta t=T / 250$. Thus, a time-step size $\Delta t=T / 500$ is adopted for the rest of modelling.

\subsection{Important performance parameters}

As fish swim freely in water, the swimming Reynolds number is an important parameter to evaluate the propulsion performance. In the present study, it is estimated using the induced time-averaged velocity $\bar{U}$ when a fish approaches a quasi-steady swimming status, the body length $L$, and water kinematic viscosity $v$ as:

$$
\operatorname{Re}=\frac{\overline{U L}}{v}
$$

For the present four cases, Re numbers are equal to $7.3 \mathrm{e} 3,1.2 \mathrm{e} 4,1.05 \mathrm{e} 4$ and $2.06 \mathrm{e} 4$, thus a laminar flow assumption is reasonably acceptable (Anderson et al., 2001).

In this study, the comparisons between rigid and flexible fins are carried out by assessing fluid force on fish body and fins, the cyclic-averaged output power $\bar{P}_{\text {out }}$, 
consumed power $\bar{P}_{c}$ and the propulsion efficiency $\eta_{\text {eff }}$. Fluid force is analysed from two aspects: cycle-averaged total force $\bar{F}_{T}$ and propulsive force $F_{P}$. These vital parameters are calculated using the following definitions:

1. Cycle-averaged total force $\bar{F}_{T}$. Total force $F_{T}$ is defined as the sum of the forces acting on the fish body $F_{B}$, dorsal fin $F_{D}$, anal fin $F_{A}$ and caudal fin $F_{C}$ as:

$$
F_{T}=F_{B}+F_{D}+F_{A}+F_{C}
$$

The cycle-averaged $\bar{F}_{T}$ for each motion cycle can illustrate the development history of the induced velocity $U$. Based on Newton's Second law, a zero $\bar{F}_{T}$ indicates a zero acceleration and thus fish swim at a cruising speed, and here we define it as a quasi-steady status.

2. Propulsive force $F_{P}$. In the present study, it is equal to the sum of forces generated by dorsal fin $F_{D}$, anal fin $F_{A}$ and caudal fin $F_{C}$ as:

$$
F_{P}=F_{D}+F_{A}+F_{C}
$$

The force acting on each single fin $\left(F_{D}, F_{A}\right.$ and $\left.F_{C}\right)$ is also analysed so that the contribution of each fin to the propulsive force can be discussed.

3. Vortex visualization. The Iso-surface is visualized with Q-criterion, which is defined as:

$$
Q=\frac{1}{2}\left(\|\Omega\|^{2}-\|\Phi\|^{2}\right)
$$

where $\Omega$ and $\Phi$ is the asymmetric and symmetric parts of the dimensionless velocity gradient, and || || stands for the matrix norm (Hunt et al., 1988).

4. Averaged output power $\bar{P}_{\text {out }}$ and consumed power $\bar{P}_{c}$. As the self-propelled pufferfish mainly moves along $\mathrm{X}$ axis, the output power $P_{\text {out }}$ is considered as the product of the propulsive force $F_{P}$ and the induced velocity $U$ for quasi-steady swimming as follows:

$$
P_{\text {out }}=F_{P} \cdot U
$$


As the deformation of fish body is not considered in the present work, the consumed power $P_{c}$ is defined in Eq. (17) as the summation of the required power that dorsal, anal and caudal fins contribute to the fish self-propulsion.

$$
P_{c}=\sum(\vec{\tau} \cdot \overrightarrow{\dot{\varphi}})
$$

For each fin, its required power is defined by the multiplication of torque $\vec{\tau}$ and the angular velocity $\overrightarrow{\dot{\varphi}}$. Torque $\tau$ is obtained by integrating the moment of pressure force along the fin rotation axis over each fin surface. For rigid fins cases, the angular velocity $\dot{\varphi}$ on each hinge is used, while for flexible fins, an averaged angular velocity $\dot{\varphi}$ along seven fin rays is adopted due to the variation of undulating amplitude for each fin ray. These seven fin rays include the leading, trailing fin rays and five other fin rays evenly distributed along the fin tips. In the experiment, Li et al. (2018a) tracked these seven fin rays to obtain the kinematic motion of one fin surface through an interpolation methodology described briefly in Fig. 1(e).

5. Propulsion efficiency $\eta_{\text {eff }}$. The propulsion efficiency is another vital metric to assess the swimming performance. In the present self-propulsion study, it is estimated as the time-averaged output power $\bar{P}_{\text {out }}$ over the averaged consumed power $\bar{P}_{c}$ :

$$
\eta_{\text {eff }}=\frac{\bar{P}_{\text {out }}}{\bar{P}_{c}}
$$

This definition, also known as the Froude efficiency (Sfakiotakis et al., 1999), has been widely used in a number of previous self-propelled studies, such as the anguilliform and carangiform fish (Borazjani and Sotiropoulos, 2010), the thunniform fish (Li et al., 2017), as well as some researches on flapping wings (Abbaspour and Ebrahimi, 2015; Zhou et al., 2016).

\section{Results and discussions}

In this study, a three-dimensional self-propelled pufferfish induced by the motions of rigid/flexible dorsal, anal and caudal fins is simulated over a total of 30 motion cycles. As listed in Table 1, Case 2 and Case 4 focus on the pufferfish with flexible fins, and their corresponding conditions with rigid fins are given in Case 1 and Case 3, respectively. Our preliminary simulation results revealed that the fish mainly swam in 
$\mathrm{X}$ translational direction. Thus, the following discussions are mainly concentrated on the analysis of swimming velocity, force in the $\mathrm{X}$ direction.

\subsection{Induced velocity $U$}

The development of velocity in the $\mathrm{X}$ direction over 30 motion cycles is presented and compared in Fig. 7. It can be seen that fish swimming follows a similar trend regardless of whether they are driven by the rigid or flexible fins, i.e. a pufferfish accelerates from motionless to a quasi-steady stage as indicated in Fig. 7. Similar development stages have also been found for other types of self-propelled BCF swimmers, such as thunniform (Li et al., 2017), carangiform (Borazjani and Sotiropoulos, 2010; Li et al., 2012) and anguilliform (Kern and Koumoutsakos, 2006). Acceleration takes about 23, 24, 18 and 23 motion cycles for Cases 1 to 4, respectively.

It is interesting to note that the fish with flexible fins swims faster than that driven by rigid ones. The time-averaged velocity $\bar{U}$ during the quasi-steady stage is summarized in Table 3 for four cases. The velocity generated by flexible fins can be up to twice as much as that with rigid fins. Evaluated against the experimental measurement (Li et al., 2018a), where the velocities of $1 \mathrm{BL} / \mathrm{s}$ and $2 \mathrm{BL} / \mathrm{s}$ were specified for a live fish associated with Case 2 and Case 4, the present CFD simulations predict a forward speed of $0.96 \mathrm{BL} / \mathrm{s}$ and $1.71 \mathrm{BL} / \mathrm{s}$, which are underestimated by about $4 \%$ and $14.5 \%$, respectively. Obviously, the consistency between CFD predicted results and experiment is very well at a low speed, but the discrepancy increases when the speed increases. A relevant previous study by Wiktorowicz et al. (2007) pointed out that fish caudal peduncle started its oscillating motion at high swimming speeds above $1 \mathrm{BL} / \mathrm{s}$, while in the present CFD model the caudal peduncle motion is excluded, which is likely the reason for the discrepancy between the experimental and numerical results at $2 \mathrm{BL} / \mathrm{s}$. It is thus suggested that the caudal peduncle motion of the pufferfish during self-propulsion may not be omitted when the swimming velocity is larger than $1 \mathrm{BL} / \mathrm{s}$. This would be considered in our near future study.

It is also observed from Fig. 7 that the cruising velocity fluctuates around a mean value after reaching the quasi-steady swimming stage. The fluctuation amplitude of the velocity for each case is $0.024 \mathrm{BL} / \mathrm{s}$ (Case 1 , rigid fins), $0.049 \mathrm{BL} / \mathrm{s}$ (Case 3, rigid fins), 0.012BL/s (Case 2, flexible fins), and 0.015BL/s (Case 4, flexible fins). For flexible 
fins, velocity fluctuation accounts for $1.25 \%$ (Case 2) and $0.8 \%$ (Case 4 ) of the mean velocity $\bar{U}$. Whereas for two rigid cases, this fluctuation increases significantly to $3.9 \%$ and 5.6\% for Cases 1 and 3, respectively. A further analysis on the difference of the fluctuation amplitude of the cruising velocity will be carried out in Section 3.2 along with the investigation on hydrodynamic forces.

The phenomenon of a fluctuating cruising velocity was also observed in the previous studies for other species of fish though the fluctuating amplitude might be different. For instance, a self-propelled zebrafish larva (carangiform swimming) reached $18 \mathrm{BL} / \mathrm{s}$ with a 11.1\% fluctuation (Li et al., 2012). In the study of Kern and Koumoutsakos (2006), the final quasi-steady velocity for a typical anguilliform swimmer was $0.33 \mathrm{BL} / \mathrm{s}$ with $2.1 \%$ fluctuation. For a thunniform fish with a rigid caudal fin, it was found that a cruising velocity of $0.278 \mathrm{BL} / \mathrm{s}$ could be achieved with a fluctuation amplitude of $0.41 \%$ (Li et al., 2017).

\subsection{Hydrodynamic force and surface pressure}

In this section, hydrodynamic forces on the fish body and its fins will be discussed in the following aspects: cycle-averaged total force $\bar{F}_{T}$, instantaneous propulsive force $F_{P}$, instantaneous force on each fin $F_{D}, F_{A}$ and $F_{C}$, force $F_{B}$ and pressure distribution on fish body. For these forces, a positive value represents a thrust force, while a negative one is an indication of resistance force.

\subsubsection{Cycle-averaged total force $\bar{F}_{T}$ and instantaneous total force $F_{T}$}

Fig. 8 (a) and (b) illustrate the cycle-averaged total force $\bar{F}_{T}$ over each single cycle within 30 periods for Cases 1-4. It is seen that $\bar{F}_{T}$ for a self-propelled pufferfish with flexible fins is larger than that with rigid fins, implying that a fish with flexible fins has a larger acceleration than its counterpart of rigid fins. In addition, it is also noted that, $\bar{F}_{T}$ decreases to zero after 23, 24, 18 and 23 motion periods for Cases 1-4, suggesting that the fish starts its cruising stage from those cycles onwards. This is in line with the duration of the acceleration stage discussed in the previous Section 3.1 on the induced velocity. For the pufferfish with flexible fins, the obtained larger acceleration plus the 
longer accelerating time leads to its larger mean swimming velocity compared to that with rigid fins.

Fig. 8 (c) shows the time history of the instantaneous total force $F_{T}$ for four cases in the 28 th to $29^{\text {th }}$ motion cycle. It is noted that with rigid fins, the amplitude of $F_{T}$ is larger than that of flexible conditions. A large force amplitude represents a large fluctuation in $F_{T}$, and this has been reflected in the fluctuating velocity as discussed in Section 3.1 as indicated in Fig. 7. This phenomenon is also in line with a previous study about a self-propelled thunniform fish by Li et al. (2017), whose results showed that a large force amplitude led to a large fluctuation on the cruising velocity.

\subsubsection{Propulsive force $F_{P}$}

The instantaneous hydrodynamic propulsive force $F_{P}$, which is the sum of forces generated by anal, dorsal and caudal fins as defined in Eq. (14), is illustrated in Fig. 9(a) to (d). With an identical kinematic motion profile being imposed on the leading fin ray for both rigid and flexible fins, the amplitude of $F_{P}$ for the fish with rigid fins is larger than that of the fish driven by flexible fins. Moreover, by comparing these two $F_{P}$ within the same time frame, it can be found that there is a phase lag between flexible and rigid fins. Although the motions of fins are driven by the leading fin ray, the trailing edge fin ray also undulates for a flexible fin, leading to a diverse surface locomotion of flexible and rigid fins, which explains the phase lag between the propulsion forces.

One important feature from Fig. 9 is that the propulsive forces $F_{P}$ associated with flexible fins are almost positive, while the rigid fins generate both positive and negative forces. This suggests that flexible fins mostly generate thrust whereas rigid fins produce not only thrust but also drag.

\subsubsection{Force on each fin: $F_{D}, F_{A}$ and $F_{C}$}

To have a better understanding on the hydrodynamic performance of fins, the instantaneous forces on each fin, i.e. dorsal $F_{D}$, anal $F_{A}$ and caudal $F_{C}$, are plotted in Fig. 9. Apart from the caudal fin, dorsal and anal fins also produce propulsive force thus 
contribute to the propulsion of fish swimming. For both rigid and flexible conditions, dorsal and anal fins have nearly equal contributions to the propulsive force, which is indicated by the amplitude of $F_{D}$ and $F_{A}$. This is consistent with the findings from previous studies (Han et al., 2016; Liu et al., 2017). Han et al. (2016) found that the mean thrust coefficient of a fish with dorsal and caudal fins was equal to that with anal and caudal fins. Liu et al. (2017) studied a full fish body-fin model, which included fish body, dorsal, anal and caudal fins. In their study, dorsal and anal fins undulated with fish body without individual motions. Results of thrust force on dorsal and anal fins illustrated that these two fins played similar roles in fish propulsion.

For rigid cases of Fig. 9 (a) and (c), the amplitude of $F_{C}$ is smaller than that of $F_{D}$ and $F_{A}$. However, for cases with flexible fins, the amplitude of $F_{C}$ is equal to (for Case 2) or larger than (for Case 4) that of dorsal and anal fins as illustrated in Fig. 9 (b) and (d). Meanwhile, compared to the rigid conditions, the amplitude of $F_{C}$ with flexible fins significantly increases, but $F_{D}$ and $F_{A}$ considerably decreases. This clearly demonstrates the important impacts of flexible fins on fish hydrodynamic performance.

In addition to the above difference in the amplitude, we also noted the phase lag among $F_{C}, F_{D}$ and $F_{A}$. It can be seen from Fig. 9 that $F_{C}$ is out of phase with $F_{D}$ and $F_{A}$ for the flexible conditions, especially for Case 4. On the contrary, these forces are always in phase with each other in the rigid cases. As the propulsive force $F_{P}$ is the sum of the forces produced by all fins, i.e. $F_{D}, F_{A}$ and $F_{C}$, the difference in the phase angle of these forces leads to a smaller fluctuation of the propulsive force $F_{P}$ for flexible fins. Moreover, although the force generated by each individual fin can be negative within a certain amount of time in one motion cycle, the phase lag between these forces results in a mostly positive $F_{P}$.

\subsubsection{Force $F_{B}$ and surface pressure on body}

Apart from the aforementioned forces on fins, Fig. 9 also displays the hydrodynamic force on the fish body $F_{B}$. As indicated in the plot, $F_{B}$ always fluctuates with an averaged negative value. In addition, similar to $F_{D}, F_{A}$ and $F_{C}$, force $F_{B}$ on the fish 
body with rigid fins also suffers larger fluctuation than with flexible ones. This can be further demonstrated from the pressure contour plots in Fig. 10 for Case 3 and 4 as examples.

Fig. 10 presents the pressure (relative to the pressure at the outlet boundary) distribution around the fish body. The selected time instants $t_{1}-t_{4}$ are also marked in Fig. 9 and correspond to the moment when $F_{B}$ reaches its maximum/minimum for rigid and flexible conditions. Given a rigid condition, in Fig. 10(a), $t_{1}=28.89 T$ is the time at which $F_{B}$ falls to its minimum and at $t_{2}=29.18 T$ it reaches its maximum. For the flexible case displayed in Fig. 10(b), $F_{B}$ reaches its minimum and maximum at $t_{3}=28.22 T$ and $t_{4}=28.91 T$, respectively.

The differences of the body pressure distribution for both fin conditions exist even though the fish body is always rigid. By comparing the results at $t_{1}$ and $t_{2}$, it reveals that the pressure on the fish body changes dramatically for the rigid condition, especially in the anterior body. Left and right views also show the differences in these two moments, which explains the large fluctuation in $F_{B}$ in Fig. 9. The uniform motion of each rigid fin perturbs a larger amount of water around the model, which could be reflected by the pressure distribution on the rigid fins and will be further analysed in Section 3.3 from aspects of vortex structure. On the contrary, the difference of the pressure distribution on the fish body between $t_{3}$ and $t_{4}$ for the flexible condition is subtle. This is in accordance with the small variation of $F_{B}$ for flexible condition in the simulation. The anterior part on the fish body suffers a relatively larger pressure than the posterior part, indicating the resistance exerted upon the fish body.

\subsection{Vortex structure}

To gain a better understanding on the formation and development of vortices, as well as the interactions between vortices generated by rigid/flexible dorsal, anal and caudal fins, the vortex structure will be discussed in detail in this section in respect to their three-dimensional and two-dimensional appearances. Examples about the formation of 3D wake structures will be firstly focused on Case 3 and 4 within the accelerating stage representing rigid and flexible conditions. Subsequently, 2D contours will be presented 
for the slices of dorsal, anal and caudal fins separately for four cases. Finally, the comparison of 3D vortex associated with Case 1-4 at a given instant will be illustrated in $\mathrm{XY}$ and $\mathrm{XZ}$ planes.

Fig. 11 presents three-dimensional wake structures for (a) Case 3 (rigid) and (b) Case 4 (flexible) as examples within one motion cycle. For both conditions, like the caudal fin, the dorsal and anal fins also create vortex rings. The locomotion of fins causes the perturbation of fluid around the fish, especially the anterior part. This leads to the variation in the distribution of body pressure and the vortices shed into the wake by different fins during the locomotion, thus propelling the fish forward. For the rigid condition in Fig. 11(a), it is noted that the vortices are mainly generated by the leading and trailing fin rays and shed off laterally. The dorsal fin is selected as an example to study the evolution of vortices. After one completed motion cycle, one separate vortex can be found on the right side of the fin and another one is being generated on its left side at $\mathrm{t} / \mathrm{T}=1.3$. From $\mathrm{t} / \mathrm{T}=1.3$ to 1.6 , the dorsal fin flaps from right to left and generates a new vortex. It merges with the previous vortex on the right side. This phenomenon of vortex merging can also be observed when the fin flaps from the left to the right from $\mathrm{t} / \mathrm{T}=1.9$ to 2.2 . Thus, the vortices formed with rigid fins are primarily shed in the lateral direction at this stage. For flexible fins as plotted in Fig. 11(b), vortices are also created by the leading and trailing fin rays. With the undulating motion of the dorsal fin, the vortices are shed off from the fin tip to the downstream of the fish, which is in contrast with the rigid fin. The difference in the wake for rigid and flexible conditions can also be found on the anal and caudal fins.

In order to have a better understanding on the interaction of vortices generated by different fins, three plane slices are presented in Fig. 12 at six selected time instants for Cases 3 and 4. A red line indicates the slice position and blue arrows show the perspective. Contours of the $\mathrm{Z}$ component of vorticity are displayed for the dorsal in Fig. 12(a) and for the anal fin in Fig. 12(b). Fig. 12(c) shows the contour plots for X vorticity for caudal fin. From these slice views, disparities in the vortices development and interaction can be obviously noted. For instance, the rigid dorsal fin creates large vortices around both its leading fin ray and the fin tip while the flexible fin mainly induces vortices near its tip. Moreover, as discussed above, the vortices indicated inside the dashed-line boxes in Fig. 12(a) clearly demonstrate their movement in lateral 
directions for the rigid fin while vortex streets mainly form in the downstream of the flexible fin. Similar results can be found for the anal fin as illustrated in Fig. 12(b). For the caudal fin in Fig. 12(c), vortices generated by the dorsal and anal fins are also noticeable as highlighted in dashed-line boxes. It is found that compared to the rigid fins where these vortices are scattered, the vortices from flexible dorsal and anal fins are much closer to those from the caudal fin. Some merged vortices in the wake could also be observed at some instants, such as (fc3) and (fc6). This implies that the flexible caudal fin is largely influenced by its upstream dorsal and anal fins and a strong interaction exists among those fins.

The vortex structure for Cases 1-4 from different views at a specific time instant of $\mathrm{t} / \mathrm{T}=5.2$ is illustrated in Fig. 13 to better demonstrate their dissimilarities during the accelerating stage. For the two rigid cases, the vortices produced by the dorsal and anal fins shed off away from caudal fin with an oblique angle $\mathbf{A}$ in XZ plane in Fig. 13(a). Another oblique angle $\mathbf{B}$ is also observed in the vertical view between two rows of vortices from the dorsal and anal fins in XY plane in Fig. 13(b). With a forward speed of $0.33 \mathrm{BL} / \mathrm{s}$ and $0.51 \mathrm{BL} / \mathrm{s}$ for Case 1 and Case 3, respectively, it is clearly seen that both angles $\mathbf{A}$ and $\mathbf{B}$ decrease as the induced swimming velocity increases. In contrast to the rigid cases, it is noted that in the near wake, a single row, made of a series of linked vortex rings, is generated by each flexible fin. Unlike the rigid fins which shed scattered vortices travelling laterally, the flexible dorsal and anal fins generate vortices that are close to each other and move in the streamwise direction. Although the aforementioned 3D wake structure is complicated, some previous studies also showed similar vortex structures using simplified models. For instance, by varying the aspect ratio, defined as the ratio of major and minor axes of an ellipse, Dong et al. (2005) found that the aspect ratio of a 3D elliptical foil had significant impact on the wake structures. Two sets of vortex rings were generated by the low aspect ratio foils and formed an oblique angle to the wake centreline in the XY plane. An increasing aspect ratio lead to these two rows merging with each other, i.e. a single wake in the streamwise direction.

With a zoom-in view in $\mathrm{XZ}$ plane, it can be found that the vortices generated by different rigid fins are more scattered in Fig. 13(c) and thus little vortex interaction is discovered between the caudal fin and the other two fins. In contrast, the vortices 
generated by the flexible caudal fin are directly affected by those shedding from flexible dorsal and anal fins. Moreover, it can be obviously observed that the vortices generated by flexible dorsal, anal and caudal fins merge in some wake regions, as mentioned previously. This phenomenon is roughly in line with the 3D view proposed by Tytell (2006) about the vortex structures in the streamwise direction, where the hairpin vortex rings generated by caudal fin were influenced by the vortices of dorsal and anal fins, and these three vortices were probably linked up. It is well accepted that the vortex topology is the reflection of the energy consumption. As scattered vortices structure indicates a dissipation of energy, the above fluid flow visualization results reveal that more energy is dissipated and consumed with rigid fins compared to flexible fins, as will be discussed in the following section.

\subsection{Power and efficiency}

Summaries on the power and efficiency for four cases are given in Table 4. With the same motion imposed on the leading fin ray, the pufferfish with rigid fins (Cases 1 and 3) requires 4.6 and 6.7 times more consumed power than the corresponding flexible fins (Cases 2 and 4). However, it only produces 59.9\% and 62.5\% of the output power, i.e. the useful power, from flexible fins. These results demonstrate that the rigid fins consume larger energy/power but generate relatively smaller useful power than the flexible fins, implying that most of the input energy is dissipated with rigid fins as discussed in the vortex structure section. Hence, a dramatic improvement in efficiency is achieved for a self-propelled pufferfish with flexible fins, as presented in Cases 2 and 4.

The efficiency for several types of swimming fish from other studies are compared in Table 5. Due to the diverse definitions about the efficiency of fish swimming, the studies that gave the Froude efficiency are compared to the present study. Meanwhile, the efficiency given in Table 5 for present study is the self-propelled pufferfish with flexible fins, i.e. the realistic pufferfish (tetradontiform). It is noted that its efficiency is lower than the thunniform fish, the swimming mode of which is widely considered to be the most efficient among the various swimming types (Roper et al., 2011). However, it is noted that with multiple fins, tetradontiform fish could achieve a relatively high efficiency, compared to anguilliform and carangiform fish. 


\section{Conclusions}

In this paper, following the experimental study of multi-fin kinematics (Li et al., 2018a), numerical simulations are carried out to investigate a self-propelled pufferfish with multiple flexible fins in an accelerating and quasi-steady swimming stages. To reveal the impacts of flexible fins on thrust generation, cases with rigid fins are also considered with comparable conditions. The locomotion of the pufferfish is solved with an inhouse developed Multi-Body Dynamics approach and the commercial CFD software package ANSYS Fluent is used to solve the fluid field.

Given an identical motion on the leading fin ray of dorsal, anal and caudal fins for rigid and flexible fins, the development of the forward swimming velocity associated with four test cases, i.e. two rigid and two flexible fins, has the same tendency as those previously studied for other types of swimmers (Borazjani and Sotiropoulos, 2010; Kern and Koumoutsakos, 2006; Li et al., 2012; Li et al., 2017). Consistent with the findings from the tethered swimming (Liu et al., 2017), we found that dorsal and anal fins also contribute to the propulsive force generation under self-propelled conditions. However, the hydrodynamic performance of the pufferfish with flexible fins is found to be distinctively improved, which is evidenced via a deep analysis on the predicted velocity, hydrodynamic force, power and efficiency.

In addition, it is also noted that, driven by flexible fins, fish can swim 1.6 2 times faster than that with rigid fins due to a larger acceleration and a longer accelerating procedure to develop compared to the rigid conditions. Meanwhile, flexible fins mostly generate positive forces with a small fluctuation, but rigid fins produce both positive and negative forces with a large oscillation amplitude. Obviously, these results support the previous findings that, a swimmer with flexible fins is more efficient than the one with rigid fins. Further analysis on the vortex structure revealed that, the vortex shedding energy can be better utilized by the fish with flexible fins, which leads to less power consumption and further results in an enhanced efficiency. Although only four cases are examined due to the limited experimental data, the numerical modelling methodology developed herein can be applied to a wide range of parametric studies for MPF swimmers under free-swimming conditions. 


\section{Acknowledgement}

Results were obtained using the EPSRC funded ARCHIE-WeSt High Performance Computer (www.archie-west.ac.uk). EPSRC grant no. EP/K000586/1. Authors would like to acknowledge Dr Jianxin Hu at Zhejiang Sci-Tech University, China for her initial development of a serial Multi-Body Dynamics tool.

\section{Supplementary movie}

Supplementary movie is available.

\section{References}

Abbaspour, M., Ebrahimi, M., 2015. Comparative numerical analysis of the flow pattern and performance of a foil in flapping and undulating oscillations. Journal of Marine Science and Technology 20 (2), 257-277.

Anderson, E.J., McGillis, W.R., Grosenbaugh, M.A., 2001. The boundary layer of swimming fish. Journal of Experimental Biology 204 (1), 81-102.

Blake, R.W., Chan, K.H.S., 2011. Biomechanics of swimming in the pufferfish Diodon holocanthus: propulsive momentum enhancement is an adaptation for thrust production in an undulatory median and paired-fin swimmer. Journal of Fish Biology 79 (7), 1774 1794.

Blidberg, D.R., 2001. The development of autonomous underwater vehicles (AUV); a brief summary, Ieee Icra.

Borazjani, I., 2013. The functional role of caudal and anal/dorsal fins during the C-start of a bluegill sunfish. Journal of Experimental Biology 216 (9), 1658-1669.

Borazjani, I., Sotiropoulos, F., 2010. On the role of form and kinematics on the hydrodynamics of self-propelled body/caudal fin swimming. Journal of Experimental Biology 213 (1), 89-107.

Borazjani, I., Sotiropoulos, F., Tytell, E.D., Lauder, G.V., 2012. Hydrodynamics of the bluegill sunfish C-start escape response: three-dimensional simulations and comparison with experimental data. The Journal of experimental biology 215 (4), 671-684.

Broering, T.M., Lian, Y.-S., 2012. The effect of phase angle and wing spacing on tandem flapping wings. Acta Mechanica Sinica 28 (6), 1557-1571.

Dong, H., Mittal, R., Bozkurttas, M., Najjar, F., 2005. Wake Structure and Performance of Finite Aspect-Ratio Flapping Foils, 43rd AIAA Aerospace Sciences Meeting and Exhibit. American Institute of Aeronautics and Astronautics, pp. AIAA 2005-0081. 
Drucker, E.G., Lauder, G.V., 2001. Locomotor function of the dorsal fin in teleost fishes: experimental analysis of wake forces in sunfish. Journal of Experimental Biology 204 (17), 2943-2958.

Gordon, M., Plaut, I., Kim, D., 1996. How puffers (Teleostei: Tetraodontidae) swim. Journal of Fish Biology 49 (2), 319-328.

Han, P., Liu, G., Ren, Y., Dong, H., 2016. Computational Analysis of 3D Fin-Fin Interaction in Fish's Steady Swimming, ASME 2016 Fluids Engineering Division Summer Meeting FEDSM2016, Washington, DC, USA, pp. FEDSM2016-7699.

$\mathrm{Hu}$, J., 2016. Numerical study on hydrodynamic performance of bio-mimetic locomotion, Naval Architecture, Ocean and Marine Engineering. University of Strathclyde, Glasgow, Scotland, UK.

Hunt, J.C., Wray, A.A., Moin, P., 1988. Eddies, streams, and convergence zones in turbulent flows, Studying Turbulence Using Numerical Simulation Databases, 2. Proceedings of the 1988 Summer Program; p. p 193-208.

Ijspeert, A.J., 2014. Biorobotics: Using robots to emulate and investigate agile locomotion. Science 346 (6206), 196-203.

Kern, S., Koumoutsakos, P., 2006. Simulations of optimized anguilliform swimming. Journal of Experimental Biology 209 (24), 4841-4857.

Kim, B., Park, S.G., Huang, W., Sung, H.J., 2016. Self-propelled heaving and pitching flexible fin in a quiescent flow. International Journal of Heat and Fluid Flow 62, 273 281.

Kourosh, S., Qiang, Z., 2015. Performance of synchronized fins in biomimetic propulsion. Bioinspiration \& biomimetics 10 (2), 026008.

Lauder, G.V., Madden, P.G.A., 2007. Fish locomotion: kinematics and hydrodynamics of flexible foil-like fins. Experiments in Fluids 43 (5), 641-653.

Li, G., Müller, U.K., van Leeuwen, J.L., Liu, H., 2012. Body dynamics and hydrodynamics of swimming fish larvae: a computational study. Journal of Experimental Biology 215 (22), 4015-4033.

Li, G., Müller, U.K., van Leeuwen, J.L., Liu, H., 2016. Fish larvae exploit edge vortices along their dorsal and ventral fin folds to propel themselves. Journal of The Royal Society Interface 13 (116).

Li, L., Li, G., Li, R., Xiao, Q., Liu, H., 2018a. Multi-fin kinematics and hydrodynamics in pufferfish steady swimming. Ocean Engineering 158, 111-122.

Li, N., Liu, H., Su, Y., 2017. Numerical study on the hydrodynamics of thunniform bioinspired swimming under self-propulsion. PloS one 12 (3), e0174740.

Li, R., Xiao, Q., Liu, Y., Hu, J., Li, L., Li, G., Liu, H., Hu, K., Wen, L., 2018b. A MultiBody Dynamics based numerical modelling tool for solving biomimetic problems. Bioinspiration and Biomimetics 13 (5), 056001. 
Lindsey, C.C., 1978. 1 - Form, Function, and Locomotory Habits in Fish, in: Hoar, W.S., Randall, D.J. (Eds.), Fish Physiology. Academic Press, pp. 1-100.

Liu, G., Ren, Y., Dong, H., Akanyeti, O., Liao, J.C., Lauder, G.V., 2017. Computational analysis of vortex dynamics and performance enhancement due to body-fin and fin-fin interactions in fish-like locomotion. Journal of Fluid Mechanics 829, 65-88.

Liu, P., Liu, Y., Huang, S., Zhao, J., Su, Y., 2018. Effects of Regular Waves on Propulsion Performance of Flexible Flapping Foil. Applied Sciences 8 (6), 934.

Park, S.G., Sung, H.J., 2018. Hydrodynamics of flexible fins propelled in tandem, diagonal, triangular and diamond configurations. Journal of Fluid Mechanics 840, 154189.

Porez, M., Boyer, F., Belkhiri, A., 2014a. A hybrid dynamic model for bio-inspired robots with soft appendages-Application to a bio-inspired flexible flapping-wing micro air vehicle, IEEE International Conference on Robotics and Automation (ICRA'2014), Hong Kong, pp. hal-00861321.

Porez, M., Boyer, F., Ijspeert, A.J., 2014b. Improved Lighthill fish swimming model for bio-inspired robots: Modeling, computational aspects and experimental comparisons. The International Journal of Robotics Research 33 (10), 1322-1341.

Roper, D.T., Sharma, S., Sutton, R., Culverhouse, P., 2011. A review of developments towards biologically inspired propulsion systems for autonomous underwater vehicles. Proceedings of the Institution of Mechanical Engineers, Part M: Journal of Engineering for the Maritime Environment 225 (2), 77-96.

Salazar, R., Fuentes, V., Abdelkefi, A., 2018. Classification of biological and bioinspired aquatic systems: A review. Ocean Engineering 148, 75-114.

Scaradozzi, D., Palmieri, G., Costa, D., Pinelli, A., 2017. BCF swimming locomotion for autonomous underwater robots: a review and a novel solution to improve control and efficiency. Ocean Engineering 130, 437-453.

Sfakiotakis, M., Lane, D.M., Davies, J.B.C., 1999. Review of fish swimming modes for aquatic locomotion. IEEE Journal of Oceanic Engineering 24 (2), 237-252.

Shoele, K., Zhu, Q., 2009. Fluid-structure interactions of skeleton-reinforced fins: performance analysis of a paired fin in lift-based propulsion. Journal of Experimental Biology 212 (16), 2679-2690.

Shoele, K., Zhu, Q., 2010. Numerical simulation of a pectoral fin during labriform swimming. Journal of Experimental Biology 213 (12), 2038-2047.

Standen, E.M., Lauder, G.V., 2007. Hydrodynamic function of dorsal and anal fins in brook trout (Salvelinus fontinalis). Journal of Experimental Biology 210 (2), 325-339.

Tytell, E.D., 2006. Median fin function in bluegill sunfish (Lepomis macrochirus): streamwise vortex structure during steady swimming. Journal of Experimental Biology 209 (8), 1516-1534. 
Wang, S., He, G., Zhang, X., 2016. Self-propulsion of flapping bodies in viscous fluids: Recent advances and perspectives. Acta Mechanica Sinica 32 (6), 980-990.

Webb, P.W., 1984. Form and function in fish swimming. Scientific American 251, $72-$ 82 .

Wiktorowicz, A.M., Lauritzen, D.V., Gordon, M.S., 2007. Powered control mechanisms contributing to dynamically stable swimming in porcupine puffers (Teleostei: Diodon holocanthus). Experiments in Fluids 43 (5), 725-735.

Xu, Y., Wan, D., 2012. Numerical simulation of fish swimming with rigid pectoral fins. Journal of Hydrodynamics, Ser. B 24 (2), 263-272.

Yu, C.-L., Ting, S.-C., Yeh, M.-K., Yang, J.-T., 2011. Three-dimensional numerical simulation of hydrodynamic interactions between pectoral-fin vortices and body undulation in a swimming fish. Physics of Fluids 23 (9), 091901.

Zhou, K., Liu, J., Chen, W., 2016. Numerical simulation of bionic foils in tandem arrangement. Advances in Mechanical Engineering 8 (5), 1687814016649299.

Zhou, K., Liu, J., Chen, W., 2017. Study on the Hydrodynamic Performance of Typical Underwater Bionic Foils with Spanwise Flexibility. Applied Sciences 7 (11), 1120.

Zhu, Q., Shoele, K., 2008. Propulsion performance of a skeleton-strengthened fin. Journal of Experimental Biology 211 (13), 2087-2100. 


\section{Figures}

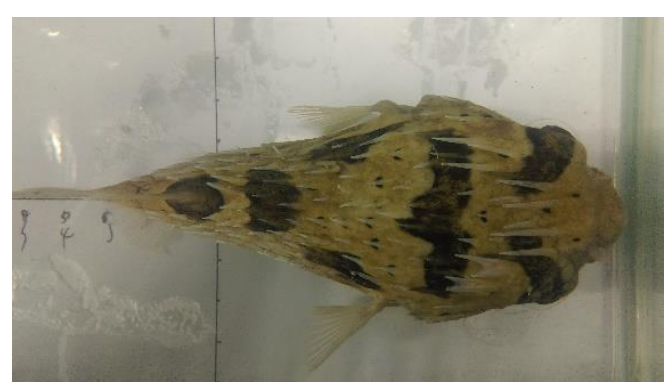

(a) Vertical view

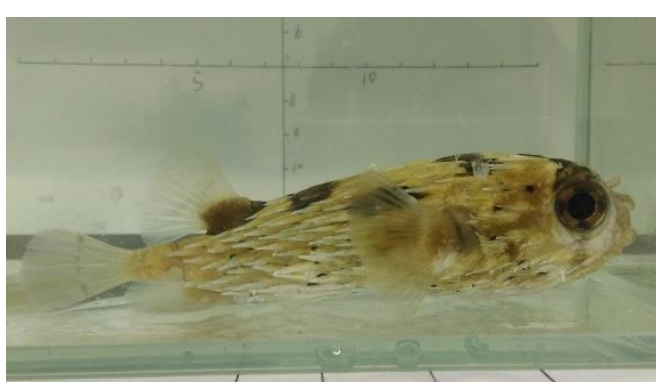

(b) Side view
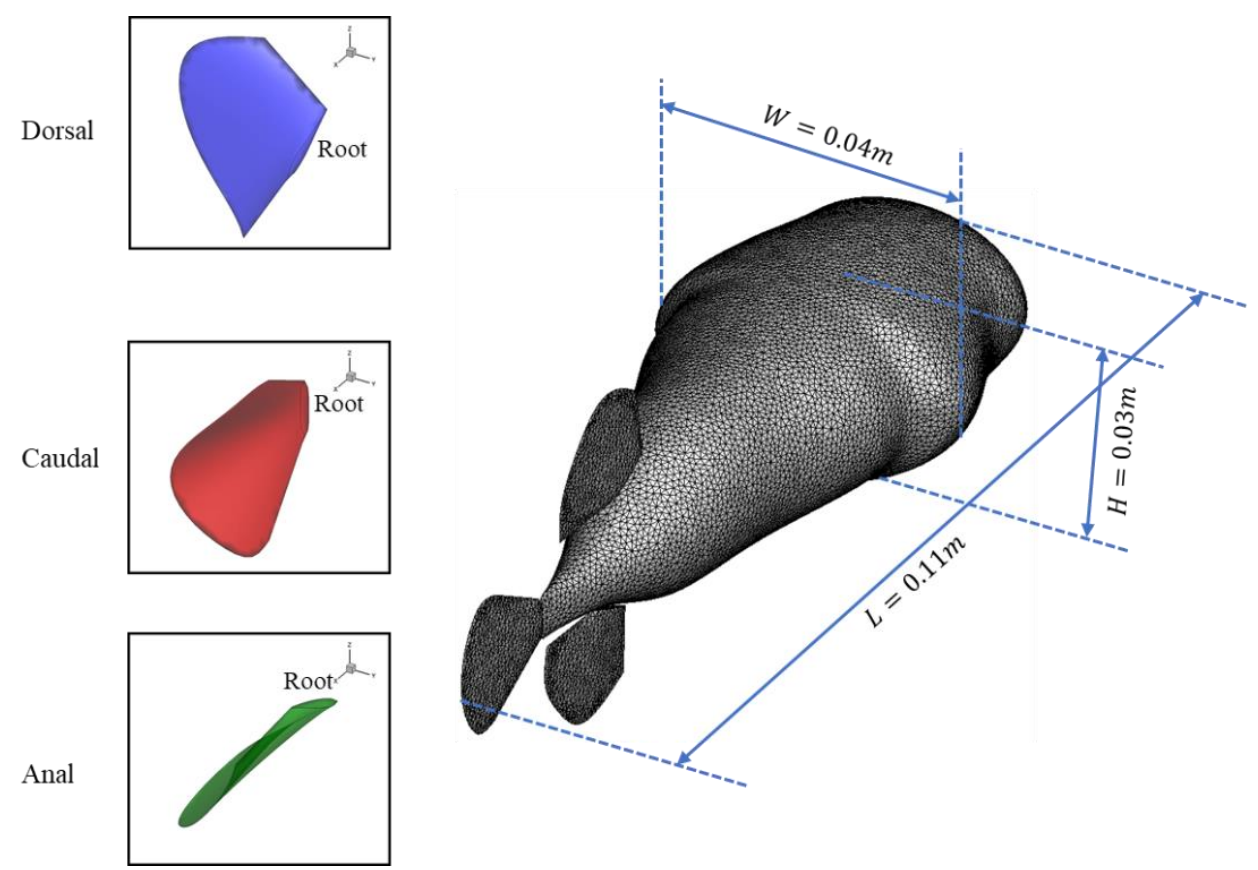

(c) Dimensions of the fish

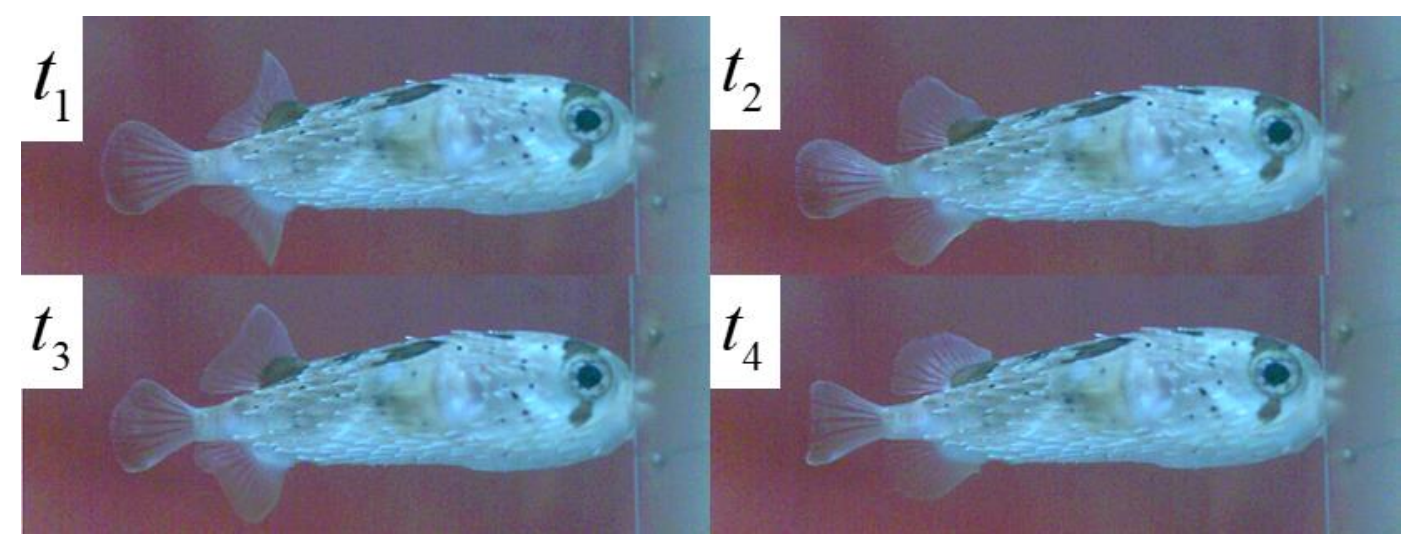

(d) Captures of four instantaneous moments in the live fish experiment 


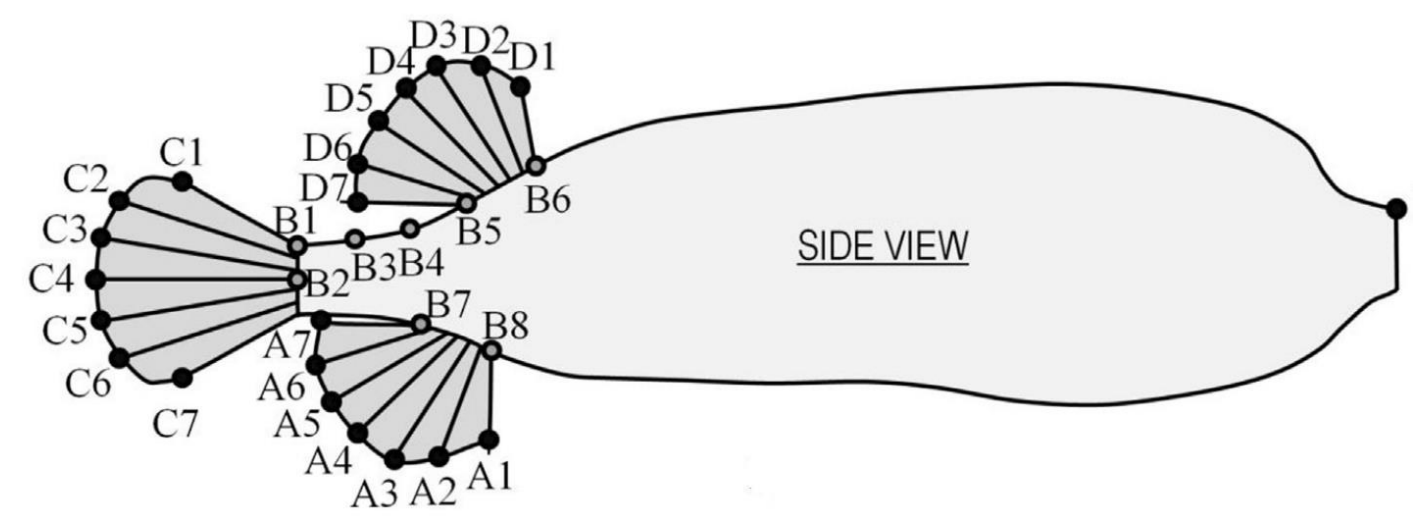

(e) Selected fin rays for dorsal (shown as D), anal (A) and caudal (C) fins used to track motion in the experiment (Li et al., 2018a)

Fig. 1 Live pufferfish in the experiment and CFD model and sketches for flexible dorsal, caudal and anal fins 
(a)

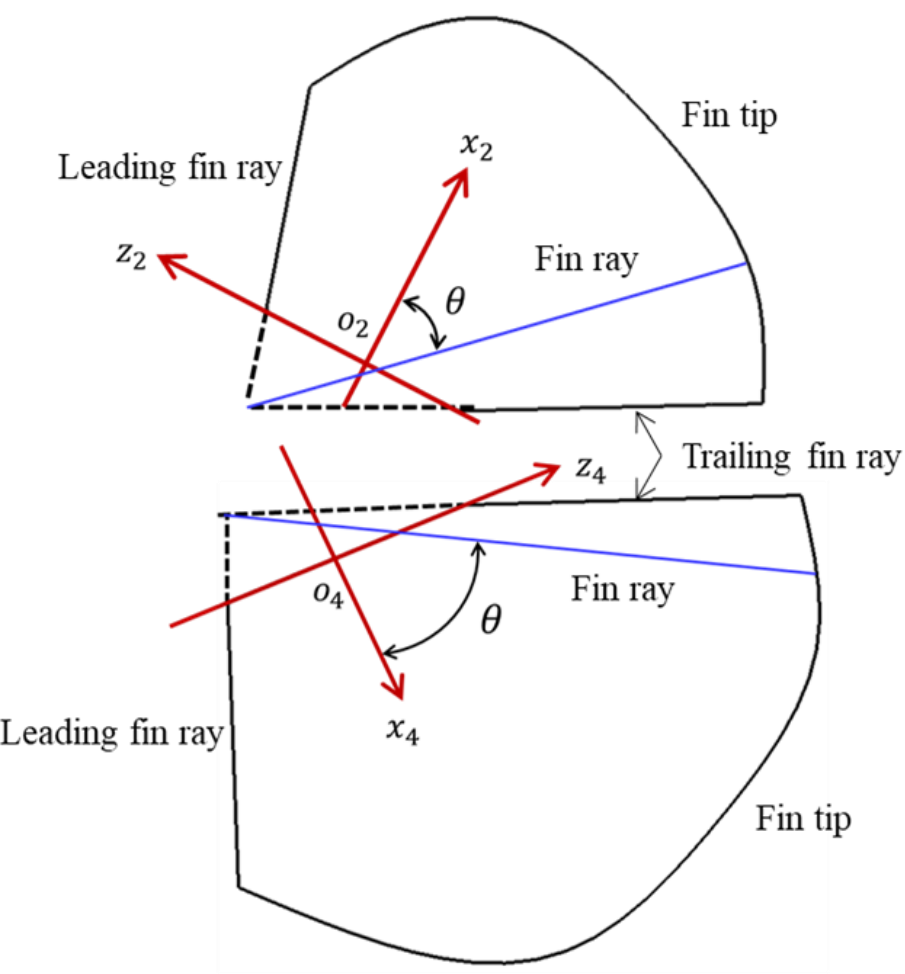

(c)

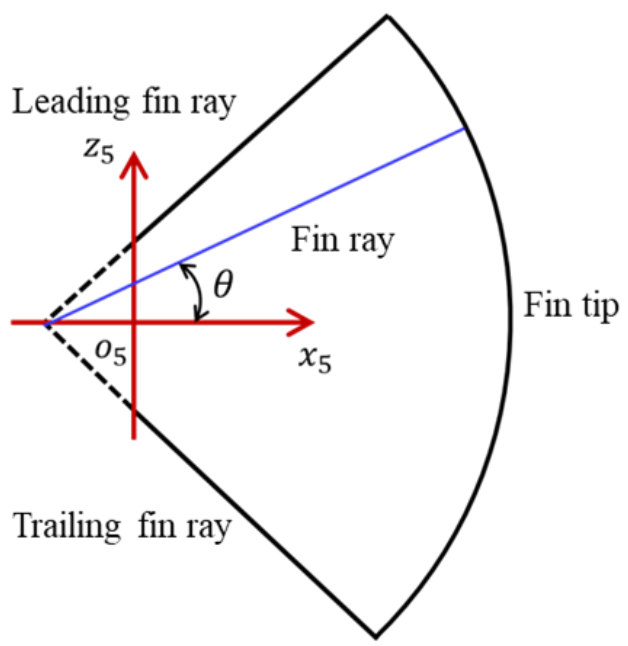

Fig. 2 Sketches for the definition of $\theta$, leading, trailing fin ray and fin tip for (a) dorsal, (b) anal and (c) caudal fins. Red lines are $\mathrm{X}$ and $\mathrm{Z}$ axis in the local coordinate for each fin, and blue line stands for a random fin ray. 

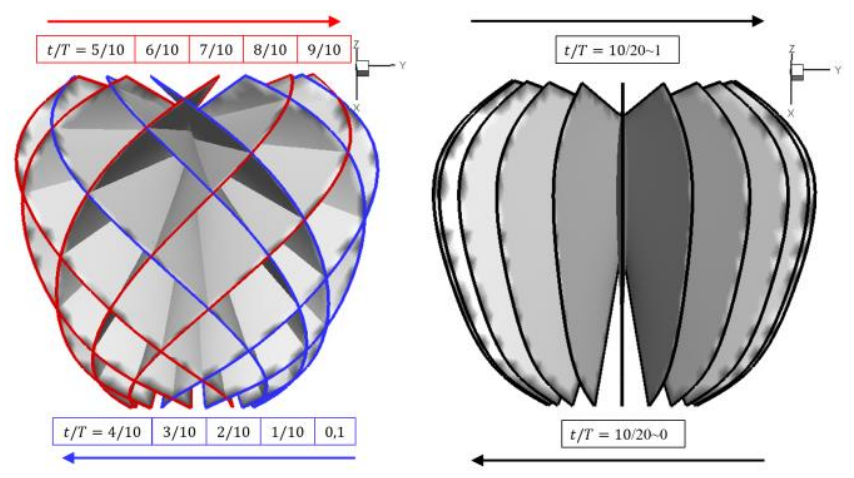

(a) Dorsal fin
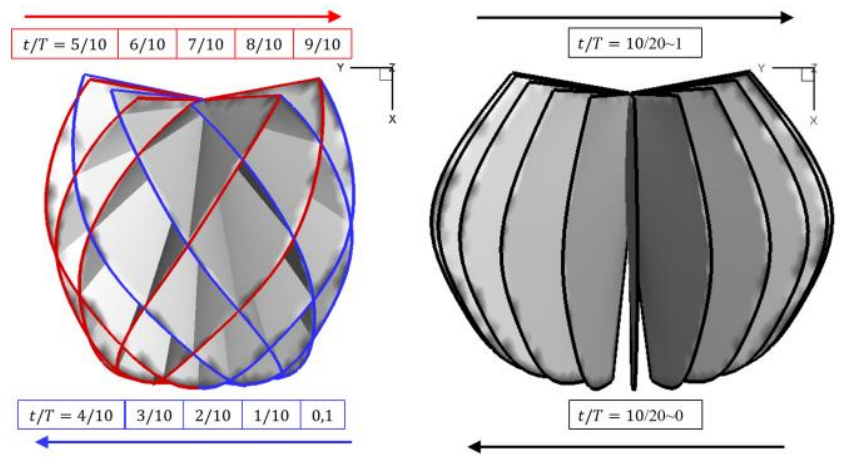

(b) Anal fin
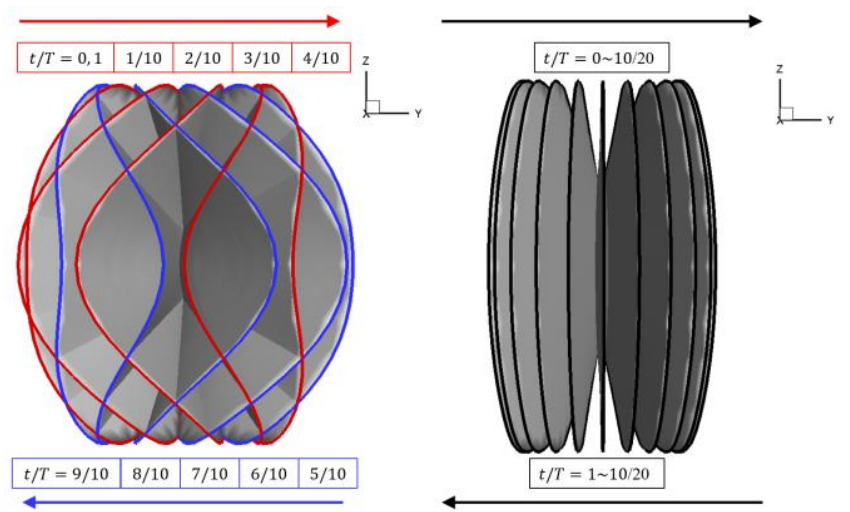

(c) Caudal fin

Fig. 3 Sketch examples for the envelopes of dorsal, anal and caudal fins in one motion period. Each envelope slice represents one motion moment. Red and blue lines in flexible condition (Case 4 left) stand for undulating from left to right and right to left respectively. Fins with rigid condition are presented at right and use Case 3 as an example. The motion period is split into 10 moments evenly for rigid condition. 


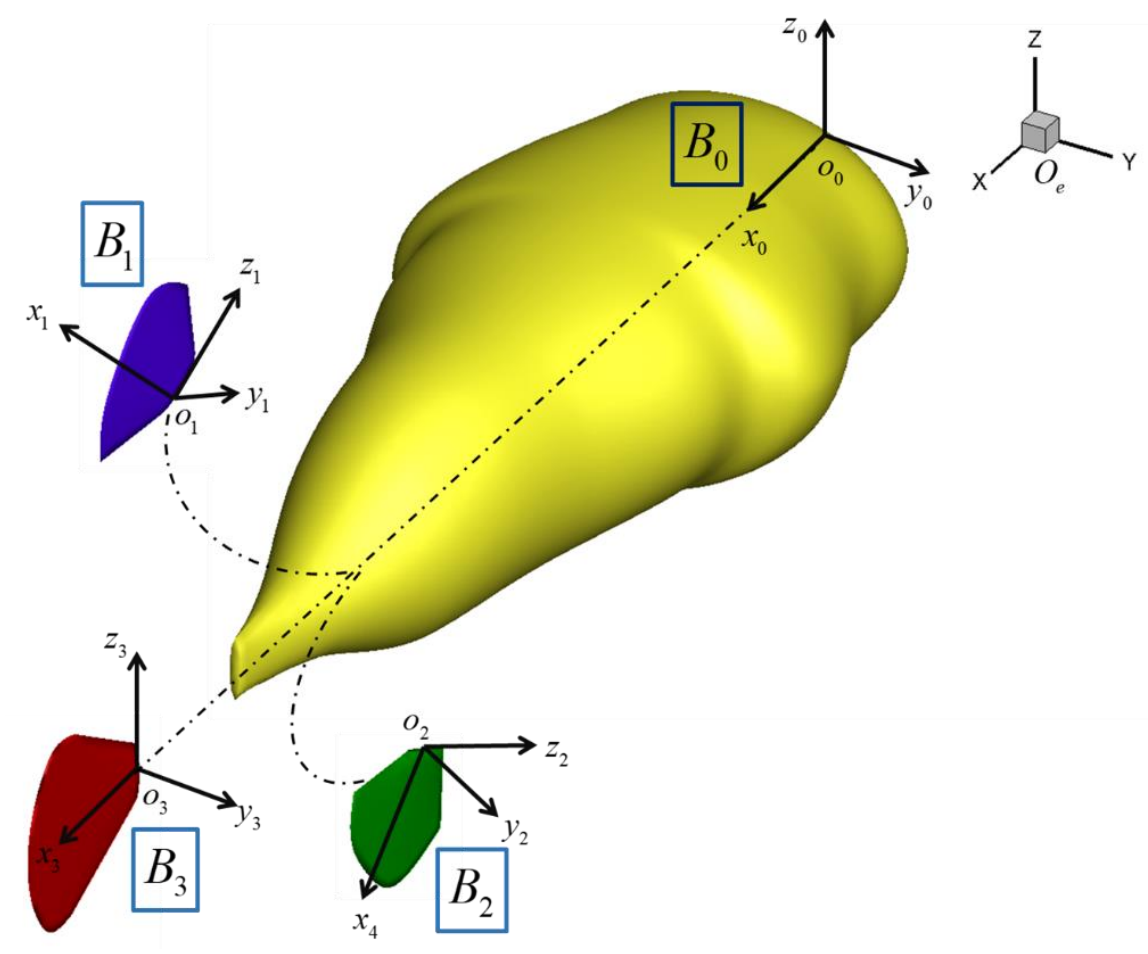

Fig. 4 Coordinate establishment of a self-propelled pufferfish, presented in the paper of Li et al. (2018b)

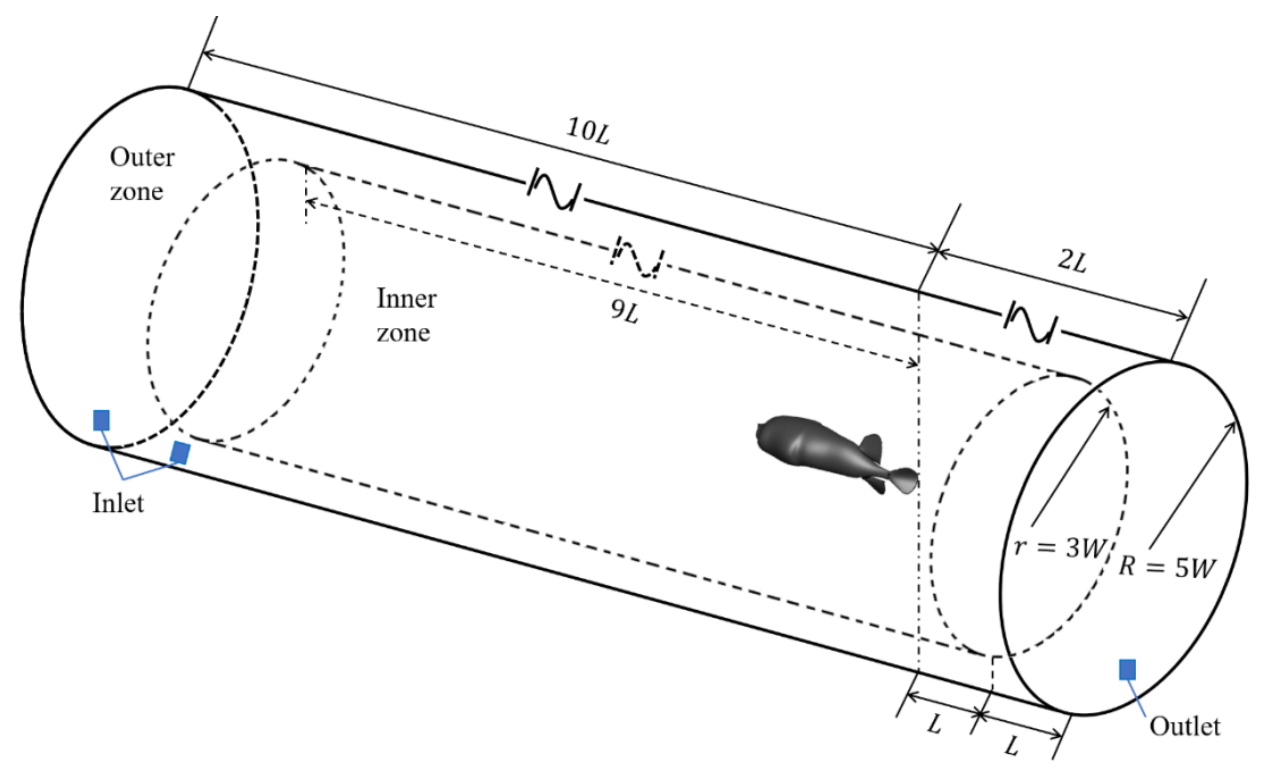

Fig. 5 Computational domain. The whole domain is split into inner and outer zone. Inner zone has finer mesh, comparing to outer zone, to better capture the flow structure of fluid field. 


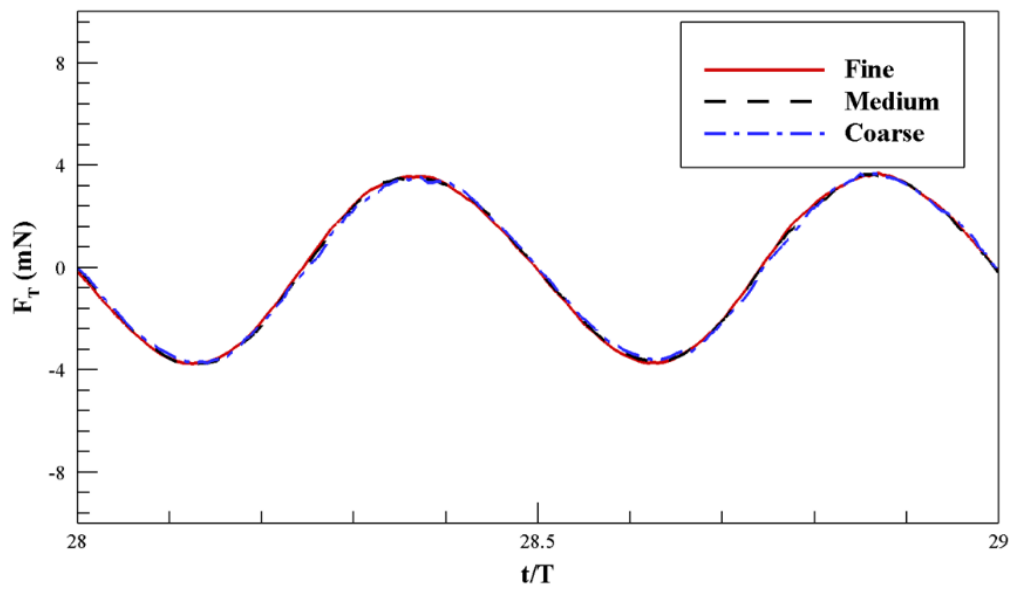

(a) Three different mesh densities for Case 4

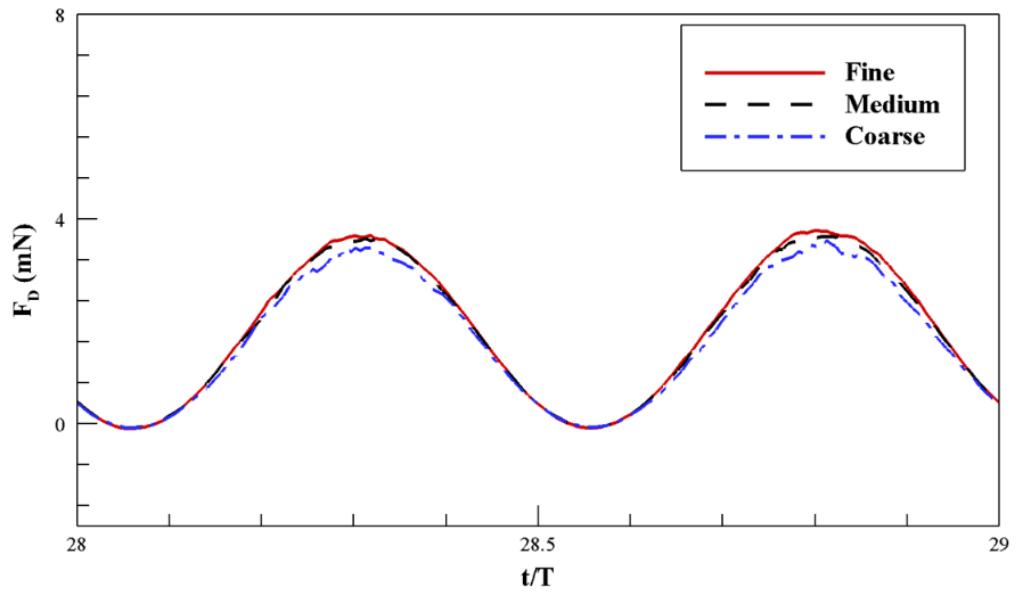

(b) Mesh density tests of force on dorsal fin for Case 4

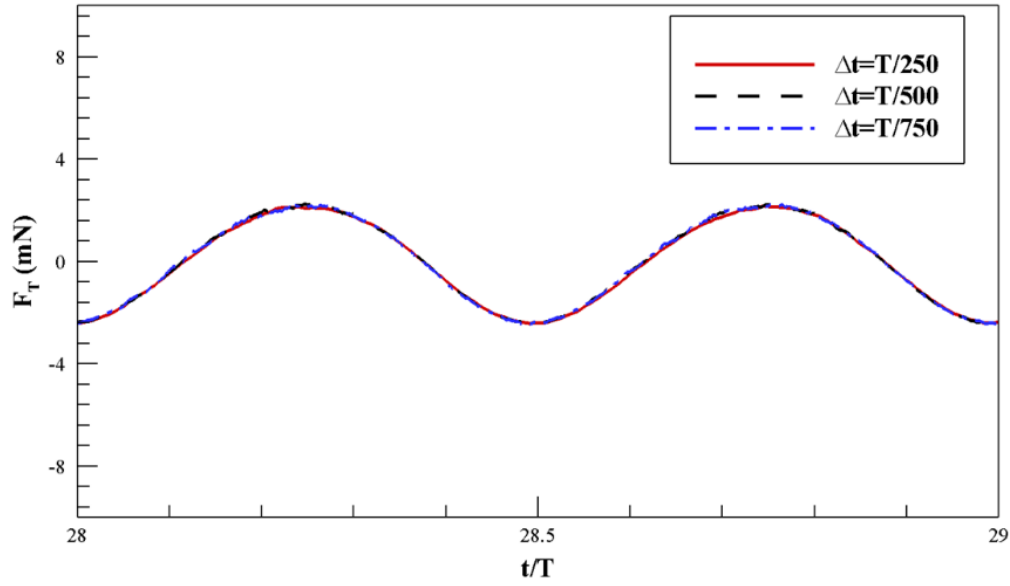

(c) Three different time-step sizes for Case 2

Fig. 6 Grid and time-step size independence tests 


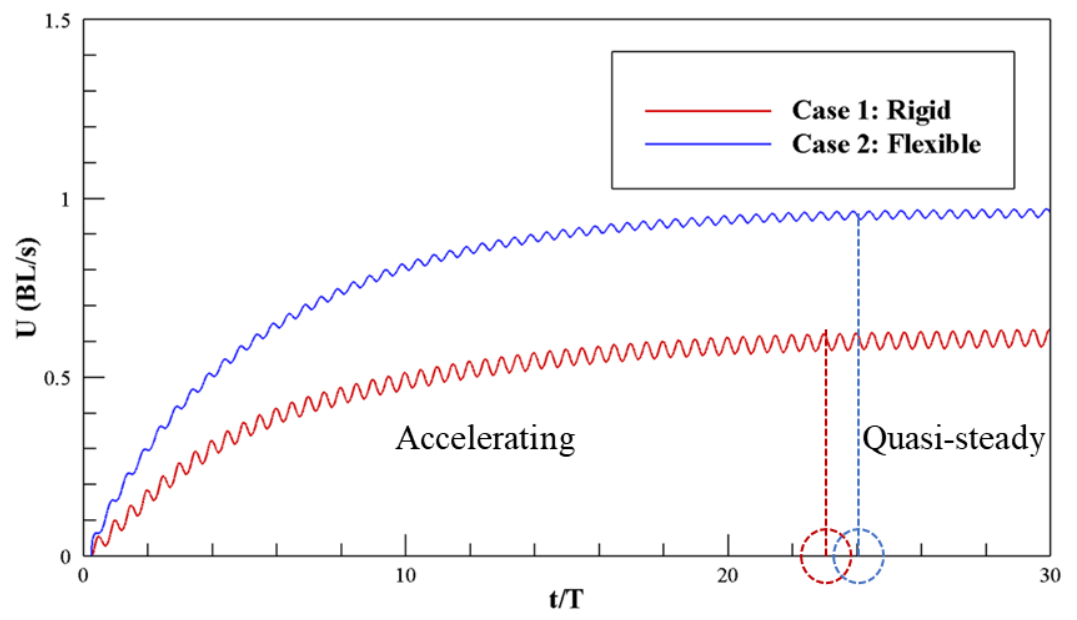

(a) Velocity development for Case 1 (rigid) and 2 (flexible)

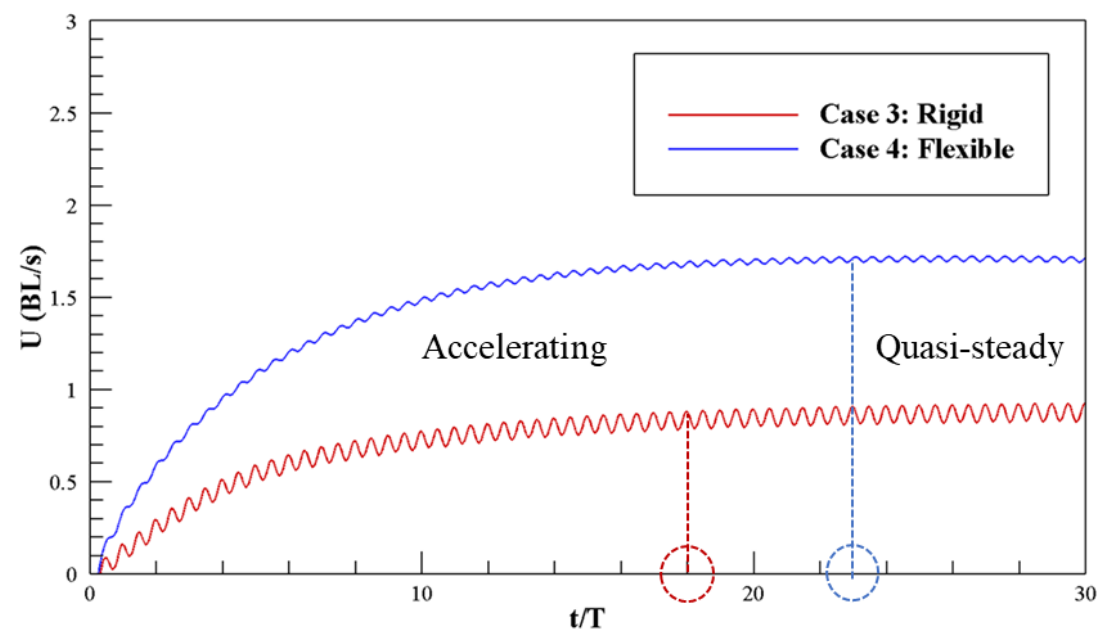

(b) Velocity development for Case 3 (rigid) and 4 (flexible)

Fig. 7 Velocity comparisons between rigid (Case 1 and 3) and flexible (Case 2 and 4) fins cases 


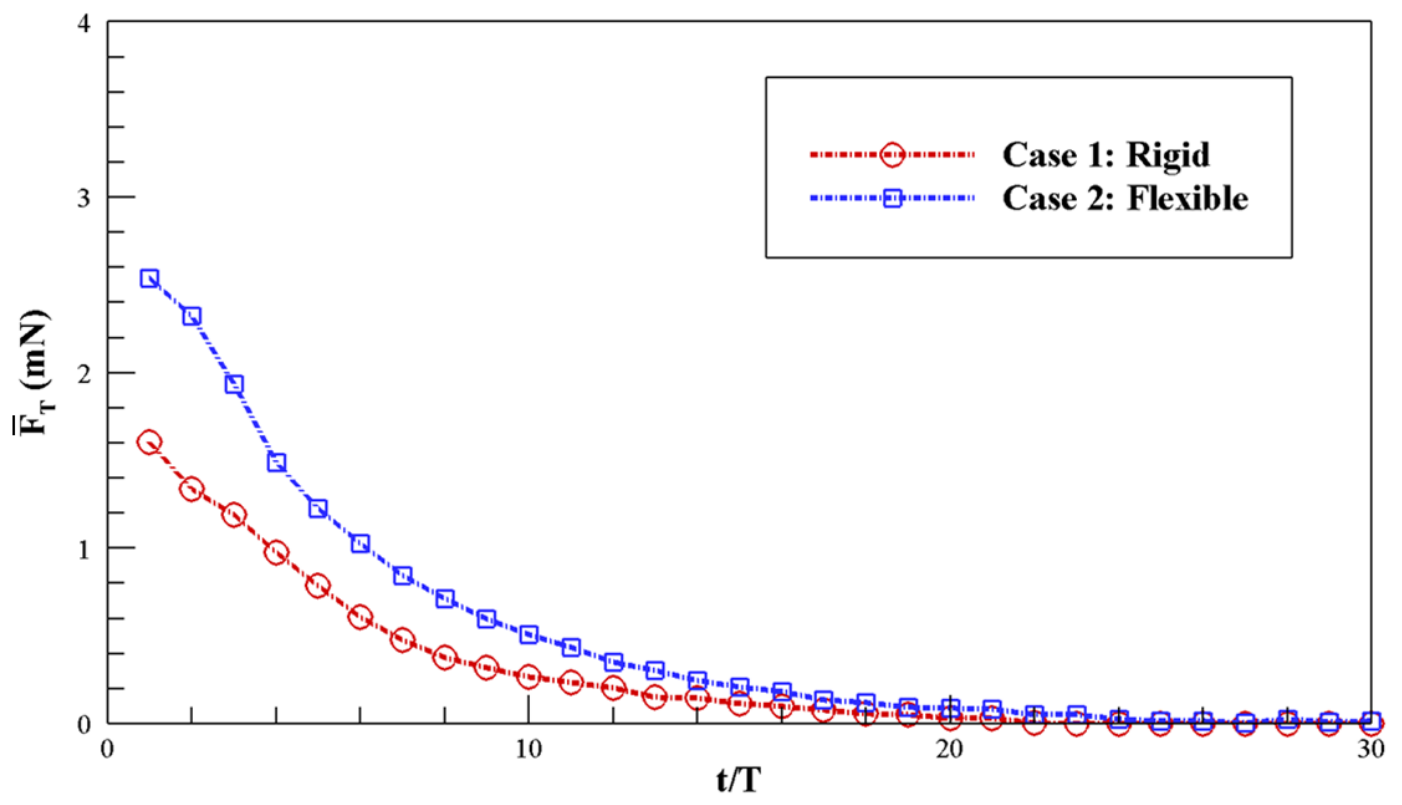

(a) Cycle-averaged total forces for Cases 1 and 2

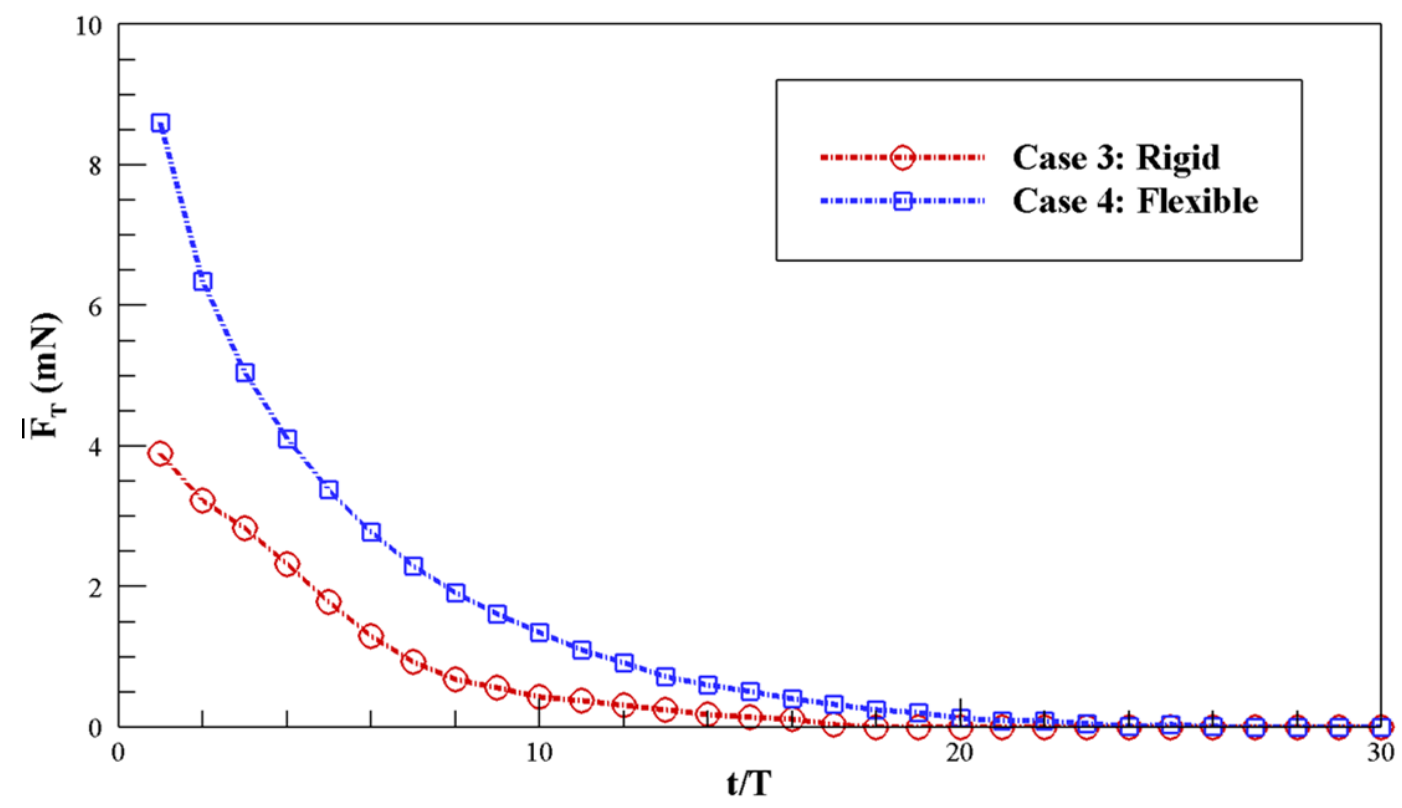

(b) Cycle-averaged total forces for Cases 3 and 4 


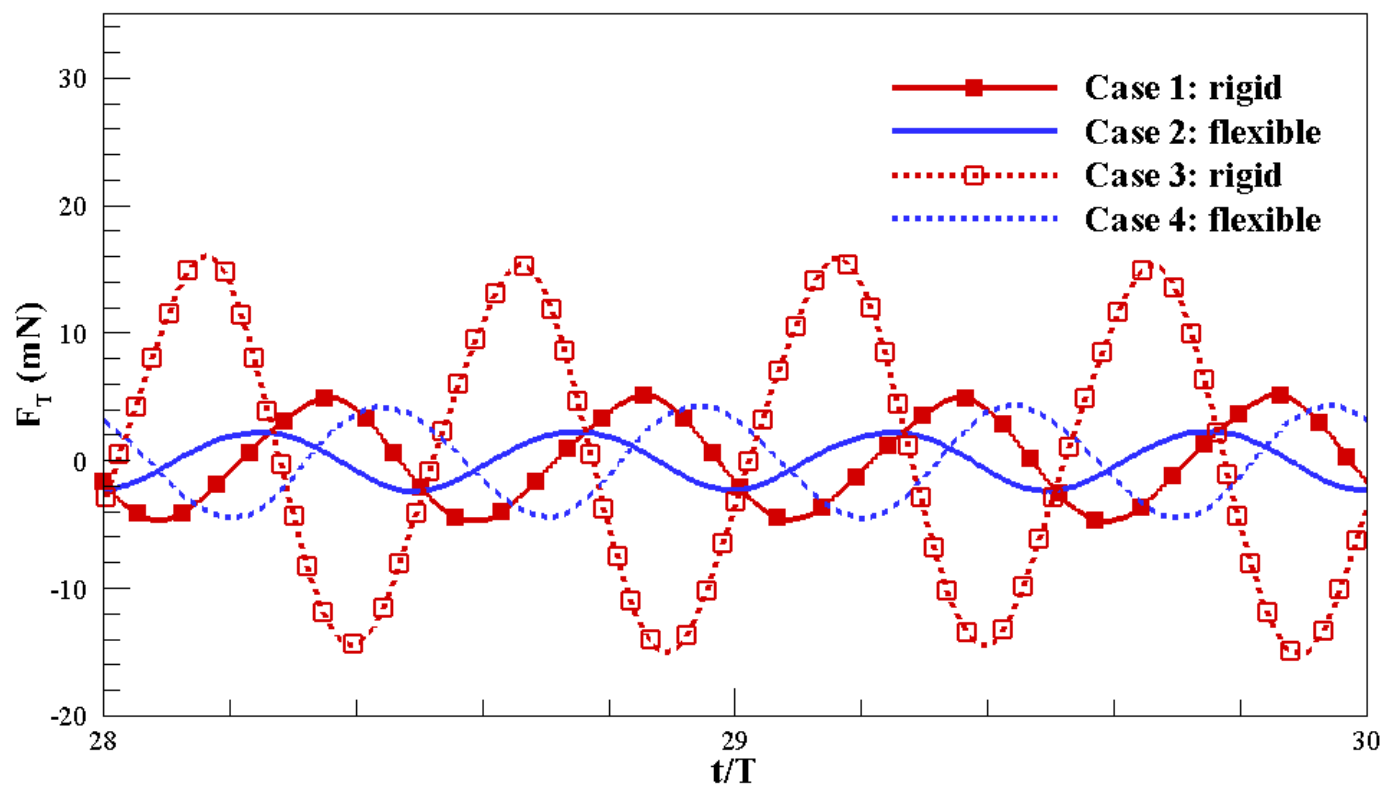

(c) Total force for four cases in two motion cycles

Fig. 8 Comparisons of Cycle-averaged total forces $\bar{F}_{T}$ and total force $F_{T}$ between rigid (Cases 1 and 3) and flexible (Cases 2 and 4) fins cases

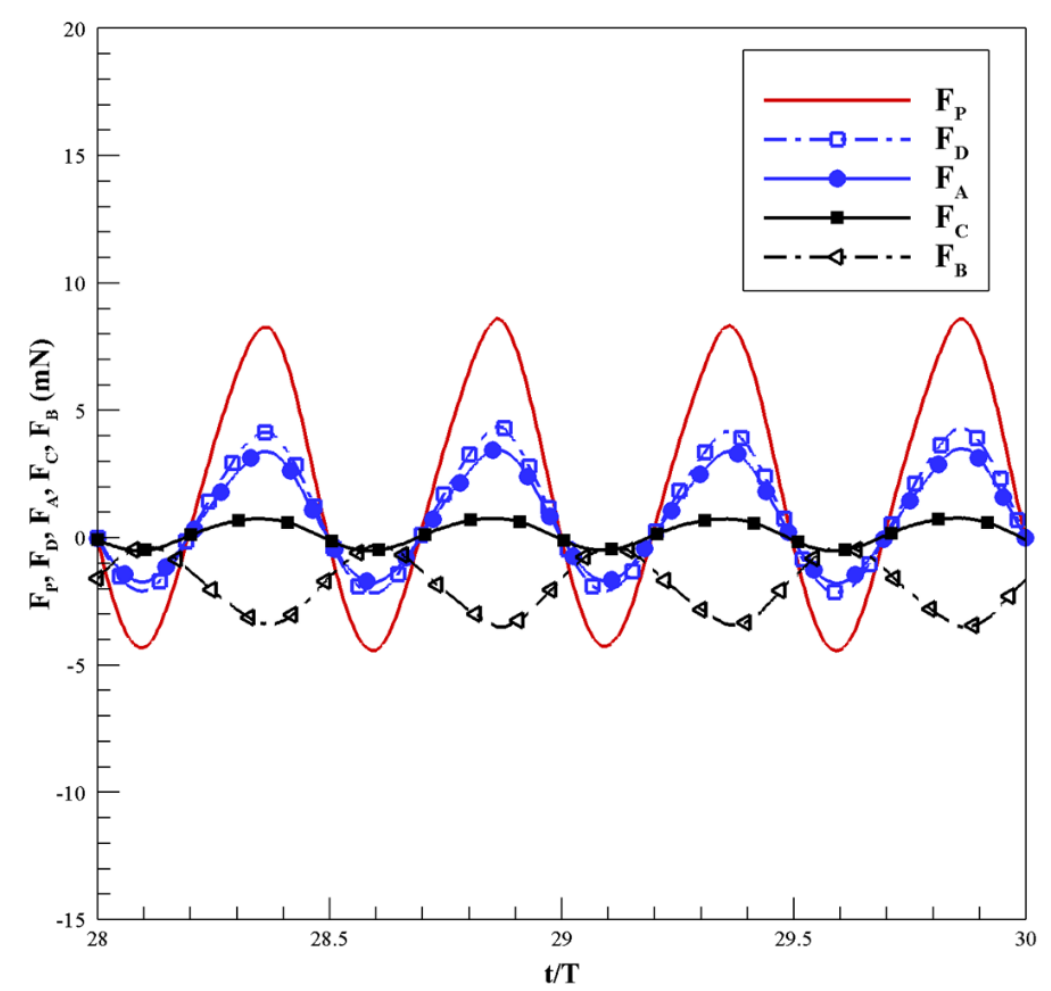

(a) Hydrodynamic forces of Case 1 (rigid fins) 


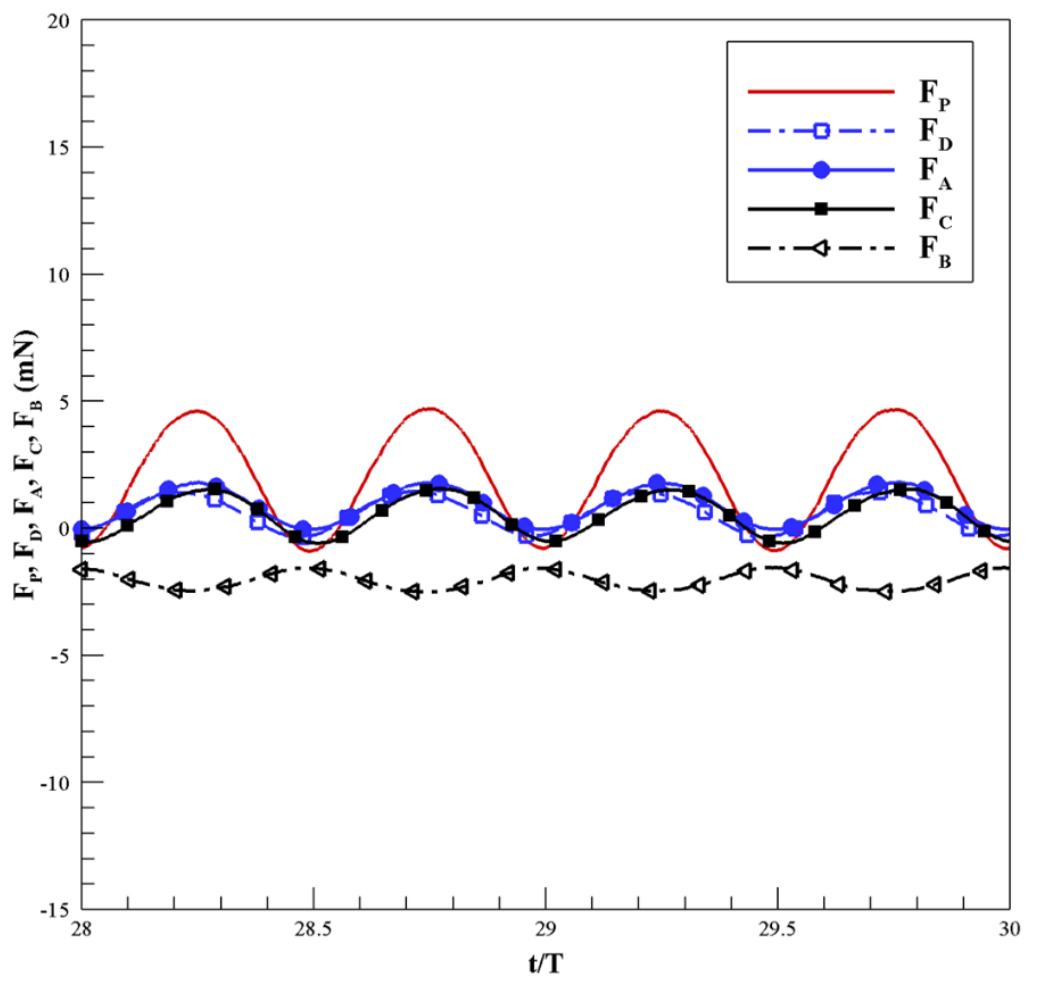

(b) Hydrodynamic forces of Case 2 (flexible fins)

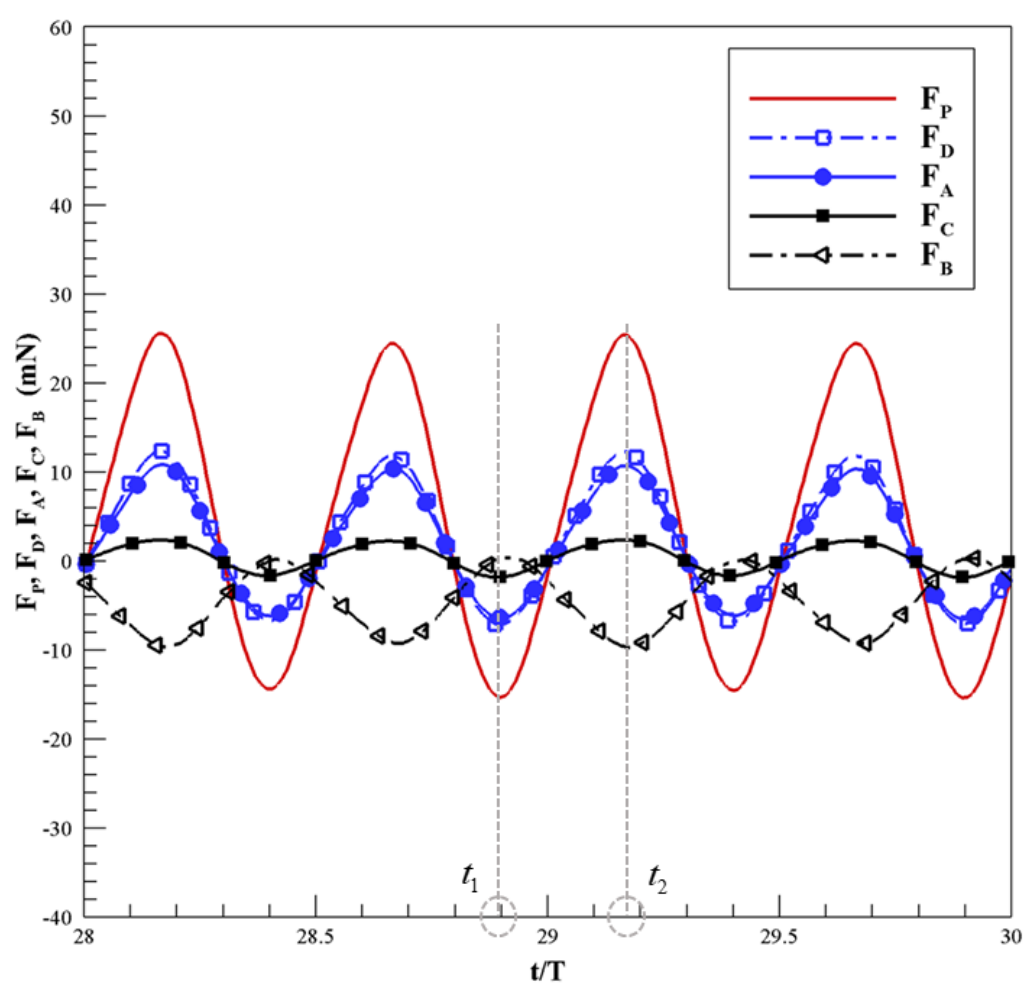

(c) Hydrodynamic forces of Case 3 (rigid fins) 


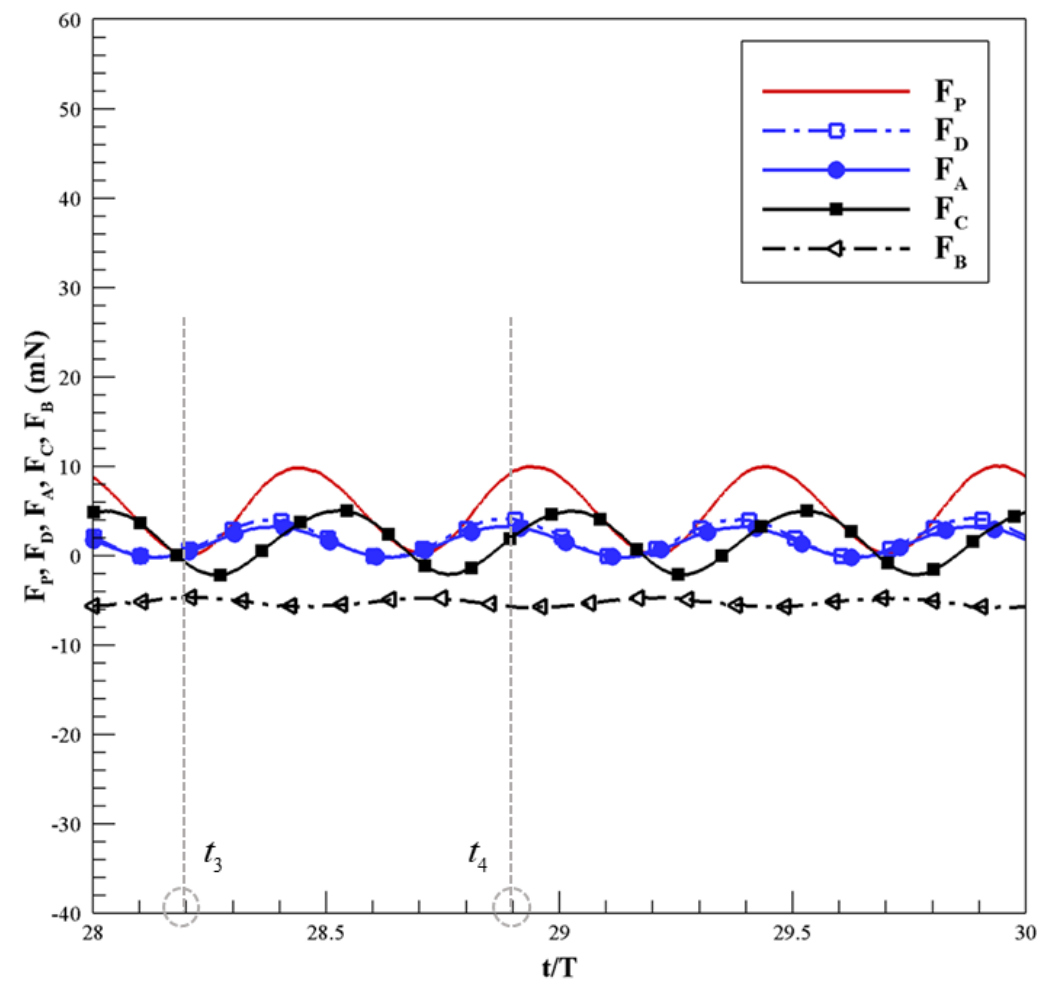

(d) Hydrodynamic forces of Case 4 (flexible fins)

Fig. 9 Hydrodynamic propulsive force $F_{P}$, force on dorsal $F_{D}$, anal $F_{A}$, caudal $F_{C}$ and body $F_{B}$ for four cases. 


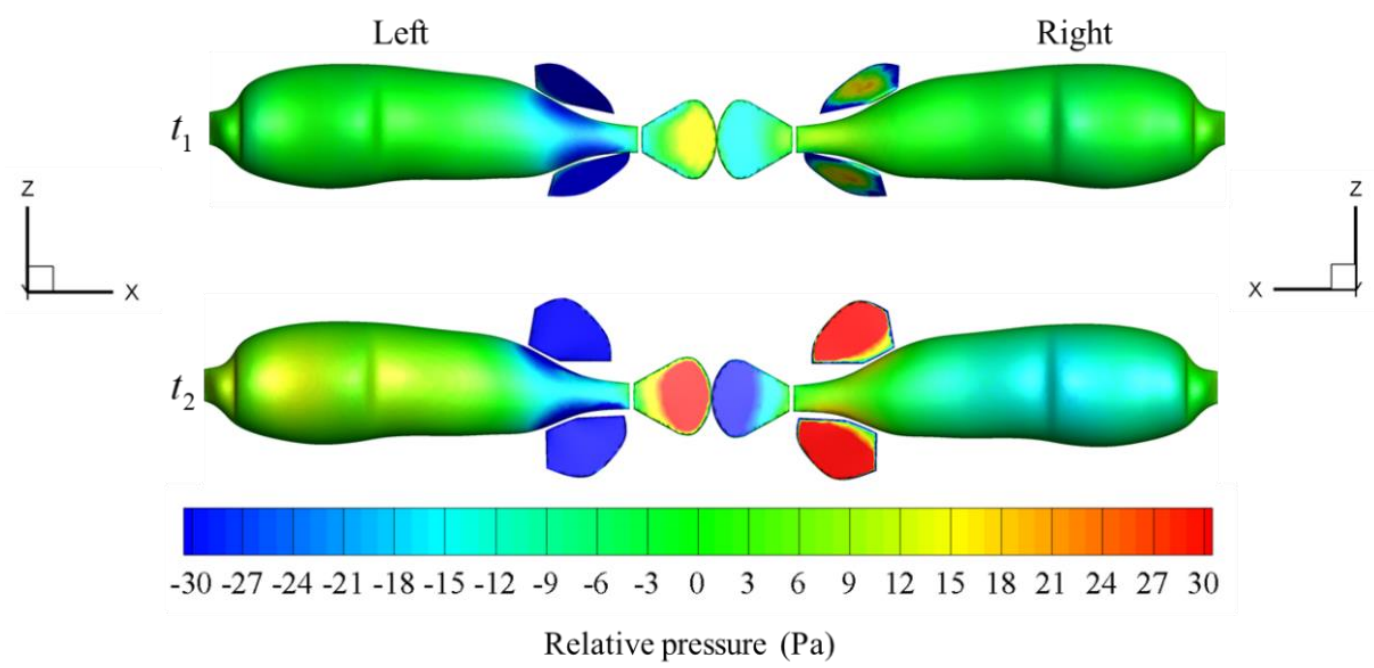

(a) Rigid fins: $t_{1}=28.89 T$ (minimum $F_{B}$ ) and $t_{2}=29.18 T$ (maximum $F_{B}$ ) for Case 3

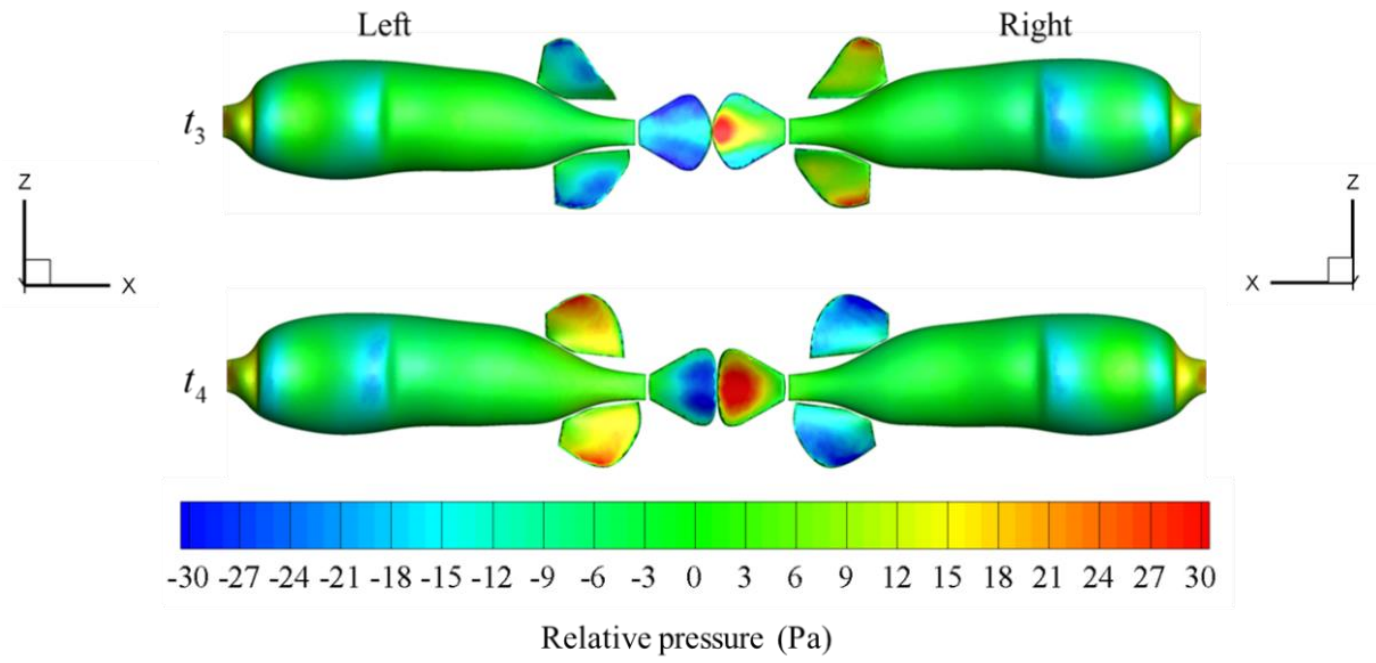

(b) Flexible fins: $t_{3}=28.22 T$ (minimum $F_{B}$ ) and $t_{4}=28.91 T\left(\right.$ maximum $F_{B}$ ) for Case 4

Fig. 10 Distribution of pressure on fish surface relative to reference pressure at outlet boundary 


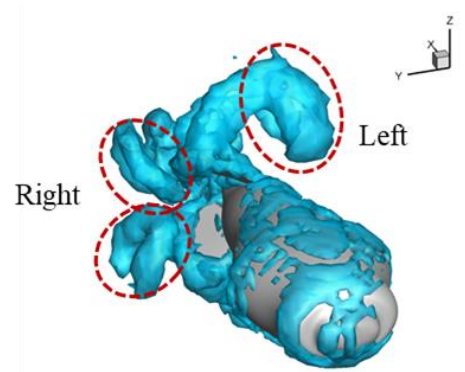

$\mathrm{t} / \mathrm{T}=1.3$

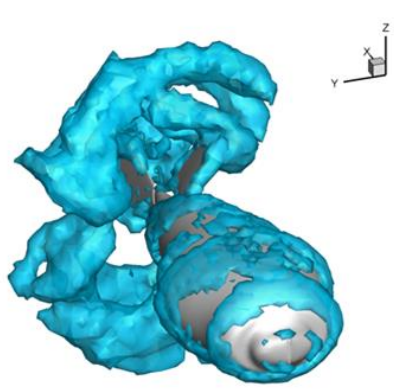

$\mathrm{t} / \mathrm{T}=1.9$
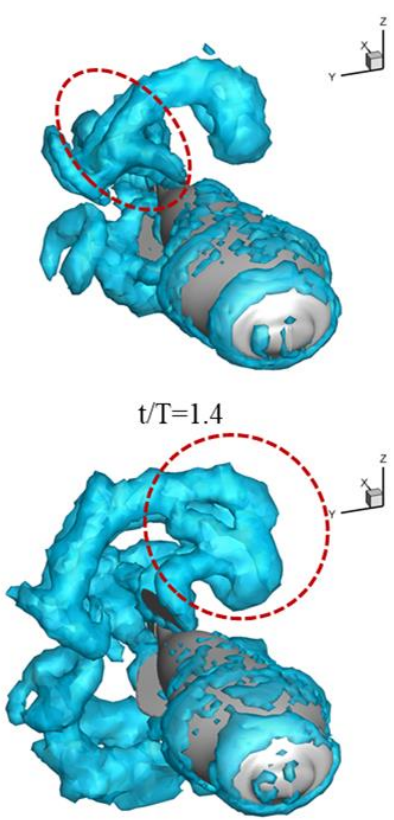

$\mathrm{t} / \mathrm{T}=2.1$

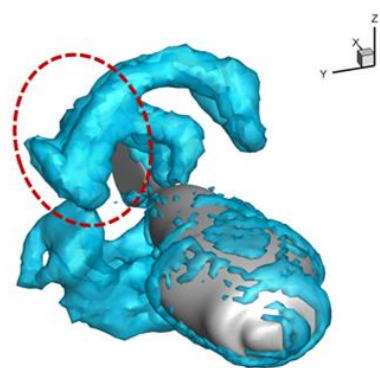

$\mathrm{t} / \mathrm{T}=1.6$

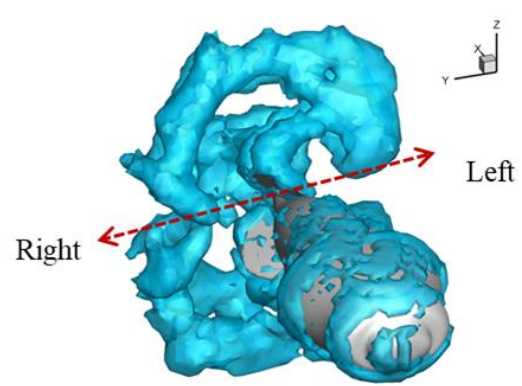

$\mathrm{t} / \mathrm{T}=2.2$

(a) Formation of vortices of pufferfish with rigid fins

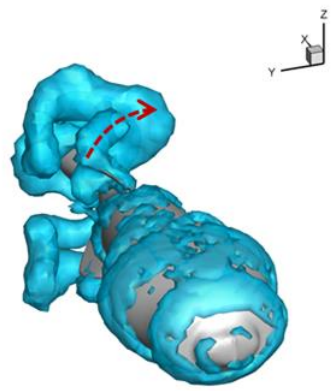

$\mathrm{t} / \mathrm{T}=1.3$

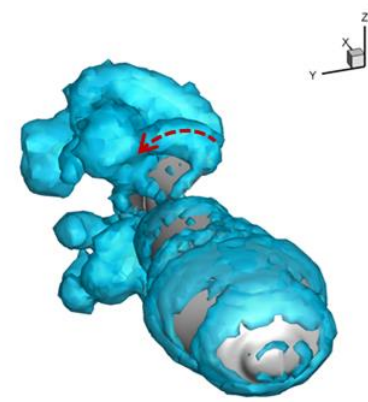

$\mathrm{t} / \mathrm{T}=1.9$

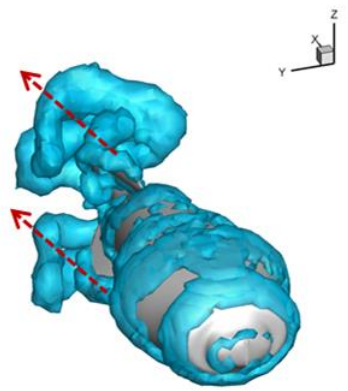

$\mathrm{t} / \mathrm{T}=1.4$

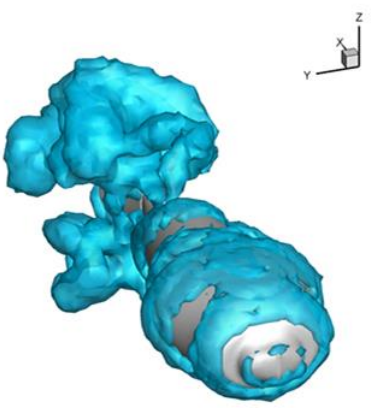

$\mathrm{t} / \mathrm{T}=2.1$

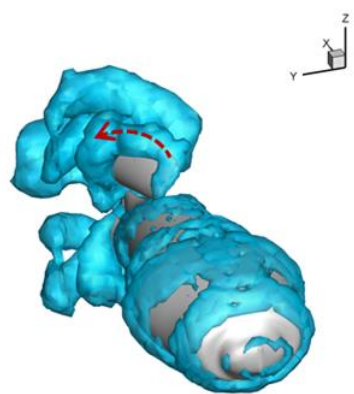

$\mathrm{t} / \mathrm{T}=1.6$

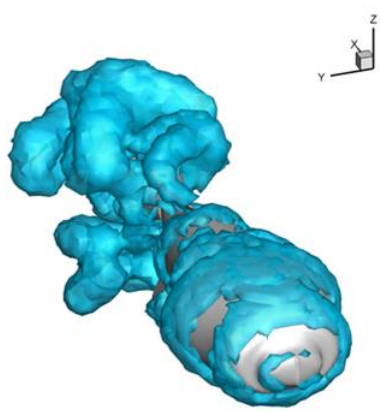

$\mathrm{t} / \mathrm{T}=2.2$

(b) Formation of vortices of pufferfish with flexible fins

Fig. 11 Vortex topology for $\mathrm{t} / \mathrm{T}=1.3-2.2$ with Iso-surface $\mathrm{Q}=2$ for (a) Case 3 and (b) Case 4 


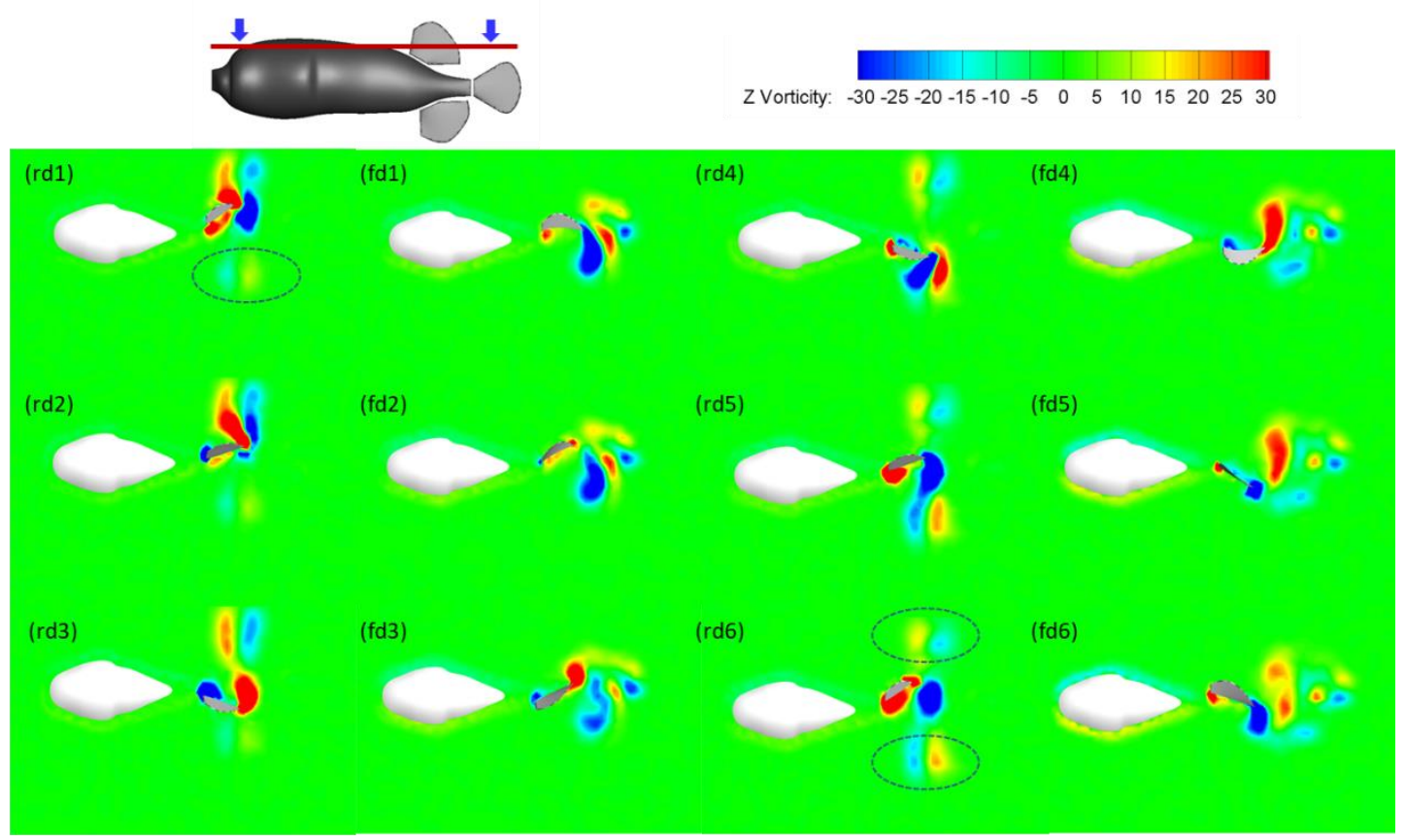

(a) Contour of z vorticity on dorsal slice. Rigid dorsal (rd) and flexible dorsal (fd)

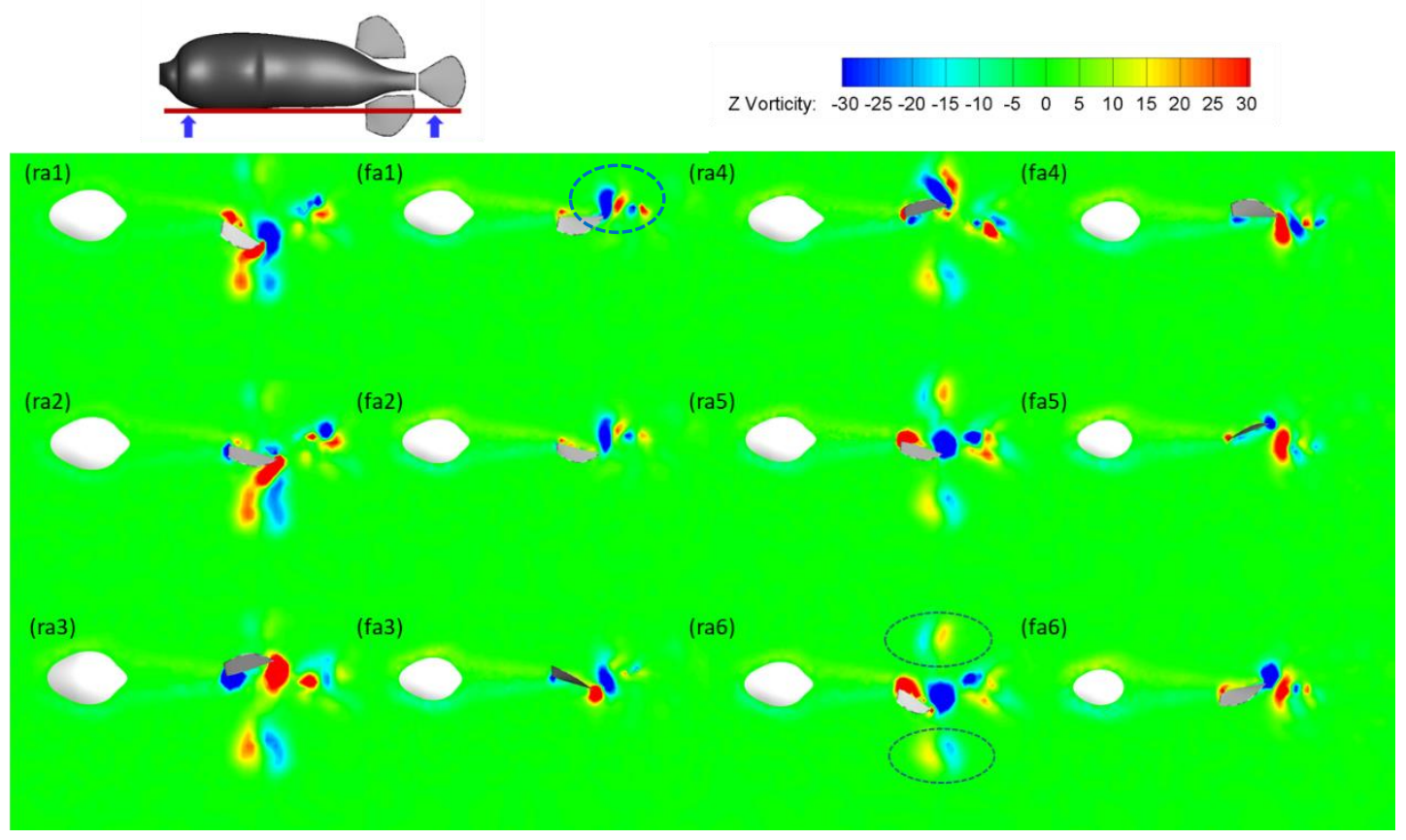

(b) Contour of z vorticity on anal slice. Rigid anal (ra) and flexible anal (fa) 


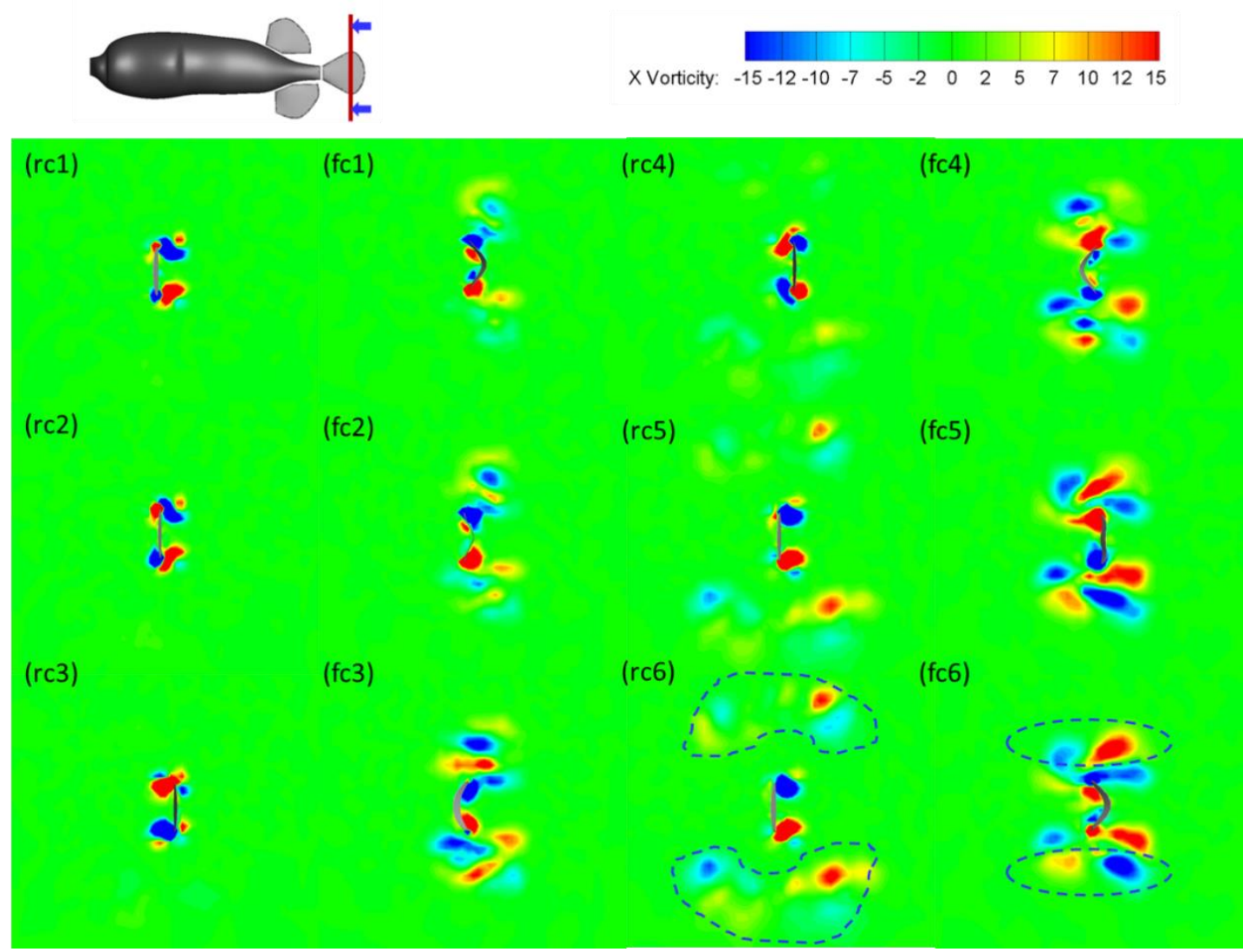

(c) Contour of x vorticity on caudal slice. Rigid caudal (rc) and flexible caudal (fc)

Fig. 12 Contours of slices for dorsal, anal and caudal fins for Cases 3 and 4 at t/T= (1) 1.3, (2) 1.4, (3) 1.6, (4) 1.9, , (5) 2.1, (6) 2.2 
(a)

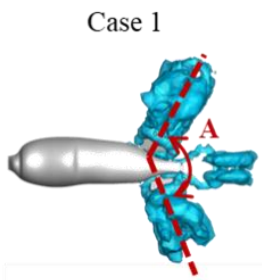

(b)

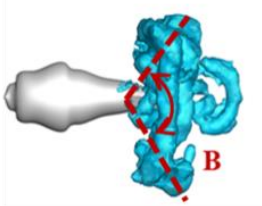

(c)

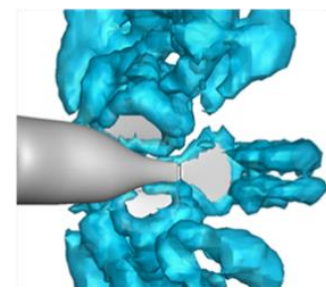

Case 2
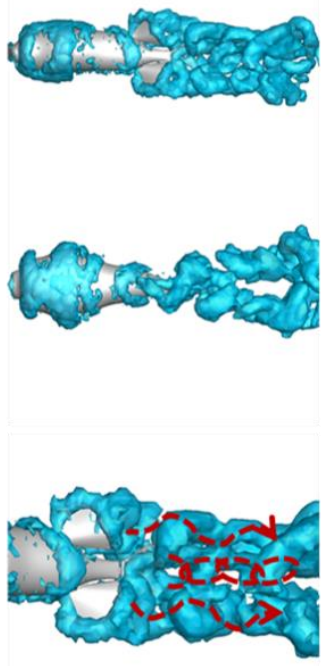

Case 3
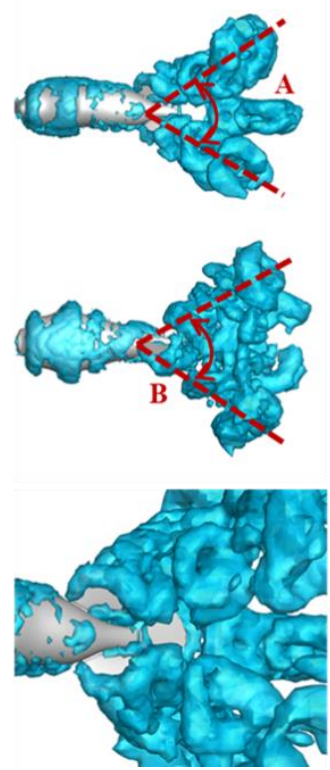

Case 4
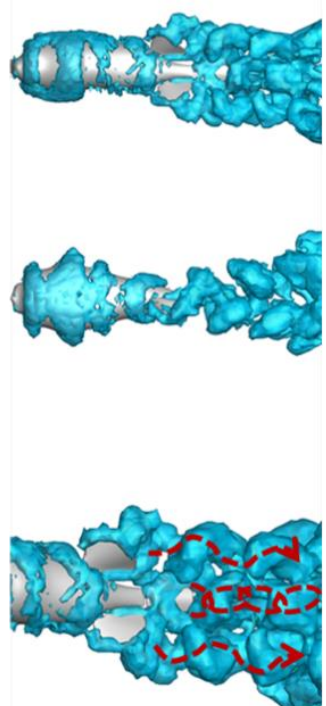

Fig. $133 \mathrm{D}$ vortex topology of Iso-surface $\mathrm{Q}=2$ at $\mathrm{t} / \mathrm{T}=5.2$ for four cases from (a) $\mathrm{XZ}$ view with included angle $\mathbf{A}$, (b) XY view with included angle $\mathbf{B}$ and (c) zoomed-in $\mathrm{XZ}$ view 
Tables

Table 1 Parameters in the kinematics equations

\begin{tabular}{|c|c|c|c|c|c|c|c|c|}
\hline \multirow{2}{*}{\multicolumn{2}{|c|}{$\begin{array}{c}\text { Case } 1,2 \\
\omega=21.4 \mathrm{rad} / \mathrm{s}\end{array}$}} & \multicolumn{3}{|c|}{ Amplitude $A$ (rad) } & \multicolumn{4}{|c|}{ Phase angle $\psi($ rad $)$} \\
\hline & & Dorsal & Anal & Caudal & & Dorsal & Anal & Caudal \\
\hline $\begin{array}{l}\text { Case } 1 \\
\text { (Rigid) }\end{array}$ & & 0.94 & 0.94 & 0.276 & & 0 & 0 & $\pi$ \\
\hline \multirow{5}{*}{$\begin{array}{c}\text { Case } 2 \\
\text { (Flexible) }\end{array}$} & $\omega_{a}$ & - & - & 6.601 & $\omega_{p}$ & - & - & 4.087 \\
\hline & $a_{I}$ & 0.378 & -0.184 & 0.357 & $p_{I}$ & -1.144 & - & 1.111 \\
\hline & $a_{I I}$ & 0.699 & 0.455 & 0.092 & $p_{I I}$ & -1.487 & -0.31 & -0.843 \\
\hline & $a_{I I I}$ & 0.264 & -0.237 & - & $p_{I I I}$ & 1.452 & -1.123 & - \\
\hline & $a_{I V}$ & 0.789 & 0.791 & - & $p_{I V}$ & 5.151 & 5.101 & - \\
\hline \multirow{2}{*}{\multicolumn{2}{|c|}{$\begin{array}{c}\text { Case } 3,4 \\
\omega=32.8 \mathrm{rad} / \mathrm{s}\end{array}$}} & \multicolumn{3}{|c|}{ Amplitude $A$ (rad) } & \multicolumn{4}{|c|}{ Phase angle $\psi($ rad $)$} \\
\hline & & Dorsal & Anal & Caudal & & Dorsal & Anal & Caudal \\
\hline $\begin{array}{l}\text { Case } 3 \\
\text { (Rigid) }\end{array}$ & & 1.03 & 1.03 & 0.31 & & 0 & 0 & $\pi$ \\
\hline \multirow{5}{*}{$\begin{array}{c}\text { Case } 4 \\
\text { (Flexible) }\end{array}$} & $\omega_{a}$ & - & - & 6.07 & $\omega_{p}$ & - & - & 3.48 \\
\hline & $a_{I}$ & 0.135 & 0.007 & 0.386 & $p_{I}$ & 0.725 & 0.725 & 0.725 \\
\hline & $a_{I I}$ & 0.32 & 0.32 & 0.32 & $p_{I I}$ & 0.265 & 0.265 & 0.265 \\
\hline & $a_{I I I}$ & 0.356 & 0.356 & - & $p_{I I I}$ & 1.473 & 1.473 & - \\
\hline & $a_{I V}$ & 0.89 & 0.89 & - & $p_{I V}$ & 4.106 & 4.106 & - \\
\hline
\end{tabular}

Table 2 Information for three different meshes

\begin{tabular}{|ccccc|}
\hline Mesh & $N_{\text {face }}$ (million) & $N_{\text {cell }}$ (million) & $\Delta h_{\min } / L$ & $\Delta h_{\max } / L$ \\
\hline Coarse & 0.013 & 0.56 & $5.2 \mathrm{e}-3$ & $4.2 \mathrm{e}-1$ \\
Medium & 0.029 & 1.59 & $3.4 \mathrm{e}-3$ & $2.9 \mathrm{e}-1$ \\
Fine & 0.051 & 3.06 & $2.9 \mathrm{e}-3$ & $2.2 \mathrm{e}-1$ \\
\hline
\end{tabular}

Table 3 Time-averaged velocity in $\mathrm{x}$ direction for quasi-steady stage

\begin{tabular}{|c|c|c|c|c|}
\hline Case No. & 1 & 2 & 3 & 4 \\
\hline $\bar{U}(B L / s)$ & 0.61 & 0.96 & 0.87 & 1.71 \\
\hline
\end{tabular}


Table 4 Averaged Power and Efficiency

\begin{tabular}{|c|c|c|c|c|}
\hline Case No. & 1 & 2 & 3 & 4 \\
\hline Averaged Consumed Power $\bar{P}_{c}(\mathrm{~mW})$ & 2.5 & 0.544 & 12 & 1.79 \\
\hline Averaged Output Power $\bar{P}_{\text {out }}(\mathrm{mW})$ & 0.13 & 0.217 & 0.5 & 0.8 \\
\hline Efficiency $\eta_{\text {eff }}$ & $5.3 \%$ & $39.82 \%$ & $3.87 \%$ & $45.44 \%$ \\
\hline
\end{tabular}

Table 5 Efficiency comparisons for different swimmers

\begin{tabular}{|c|c|c|}
\hline Literature & Fish & Efficiency \\
\hline Li et al. (2017) & $\begin{array}{c}\text { Tuna } \\
\text { (Thunniform) }\end{array}$ & $30-65 \%$ \\
\hline Present study & $\begin{array}{c}\text { Pufferfish } \\
\text { (Tetradontiform) }\end{array}$ & $39 \%-45 \%$ \\
\hline Borazjani and Sotiropoulos (2010) & $\begin{array}{c}\text { Eel } \\
\text { (Anguilliform) }\end{array}$ & $17.5 \%-32.1 \%$ \\
\hline Borazjani and Sotiropoulos (2010) & $\begin{array}{c}\text { Mackerel } \\
\text { (Carangiform) }\end{array}$ & $18.6 \%-22.1 \%$ \\
\hline
\end{tabular}

